\title{
Perspective
}

PERSPECTIVE Actualité en histoire de l'art

1 | 2018

Actualité en histoire de l'art

\section{Art dégénéré et spoliations des Juifs durant la Seconde Guerre mondiale}

Un débat entre Arno Gisinger, Emmanuelle Polack, Juliette Trey et Christoph Zuschlag, conduit par Johann Chapoutot

Arno Gisinger, Emmanuelle Polack, Juliette Trey, Christoph Zuschlag et Johann Chapoutot

\section{OpenEdition}

Journals

Édition électronique

URL : http://journals.openedition.org/perspective/8968

DOI : $10.4000 /$ perspective.8968

ISSN : 2269-7721

Éditeur

Institut national d'histoire de l'art

Édition imprimée

Date de publication : 30 juin 2018

Pagination : 13-36

ISBN : 978-2-917902-46-2

ISSN : $1777-7852$

Référence électronique

Arno Gisinger, Emmanuelle Polack, Juliette Trey, Christoph Zuschlag et Johann Chapoutot, « Art

dégénéré et spoliations des Juifs durant la Seconde Guerre mondiale », Perspective [En ligne], 1 | 2018, mis en ligne le 28 novembre 2018, consulté le 10 décembre 2020. URL : http://

journals.openedition.org/perspective/8968; DOI : https://doi.org/10.4000/perspective.8968 


\section{DÉBATS}

\section{Art dégénéré et spoliations des Juifs durant la Seconde Guerre mondiale}

\author{
Un débat entre Arno Gisinger, Emmanuelle \\ Polack, Juliette Trey et Christoph Zuschlag, \\ conduit par Johann Chapoutot
}

La dégénérescence fut une des grandes angoisses de l'Occident raciste et social-darwiniste. Le développement de la médecine et des sciences naturelles, le succès foudroyant de Darwin et la transposition des concepts darwiniens de la nature à la culture, de la faune à l'homme, mais aussi la confrontation avec des mondes extra-européens qui inquiétaient autant qu'ils fascinaient, puis le drame démographique de la (Très) Grande Guerre, amenèrent les grandes nations d'Europe et d'extrême-Europe (États-Unis, Commonwealth) à se tâter le pouls : et si la "race blanche", moins primitive, animale, agressive que d'autres, était vouée à l'extinction? Et si elle avait fait son temps? Depuis que les naturalistes européens ramassaient des fossiles, on ne comptait plus les espèces, parfois gigantesques, titanesques même, qui avaient disparu. L'histoire, cimetière des géants - la biologie, arène des forts, tribunal des faibles.

Les "peuples primitifs" furent parfois considérés comme une sévère alerte: le noir, presque animal, proche de sa naissance, donc de la nature, tendait un miroir au blanc décadent, efféminé, souffreteux, à ces "pâles esthètes " auxquels Hitler, dans Mein Kampf, dit préférer n'importe quelle brute analphabète, mais vigoureuse et saine.

La Grande Guerre acheva de faire paniquer les Occidentaux : la saignée démographique était un désastre quantitatif, mais aussi qualitatif - les meilleurs s'étant portés en première ligne, seuls restaient les plus faibles, les ratés, les tarés, les médiocres.

Dans les années 1920, la "dégénérescence "fut à nouveau, et plus que jamais, à l'ordre du jour - médical, eugéniste, politique. Les nazis n'inventèrent ni le mot, ni la chose, mais ils surent faire de la dégénérescence un épouvantail et de la régénérescence un programme. Tout, à leurs yeux, faisait signe vers la submersion des bons et des sains par les mauvais et les malades. Les symptômes de la dégénérescence biologique de l'Allemagne étaient partout : baisse de la natalité, soin accru aux malades par une politique sociale et sanitaire absurde, homosexualité et onanisme croissants, mélanges raciaux en hausse, perte de l'instinct racial, etc. Le symptôme le plus spectaculaire - et le plus pédagogique - était l'art dégénéré. Dégénéré et ent-artet veulent dire exactement la même chose: l'individu ou l'art dégénéré est l'individu ou l'cuvre déchu(e) de sa race, éloigné de sa biologie, étranger au sang et à l'instinct le plus sain de la race. L'art dégénéré nous donne à voir des réalités absurdes et inquiétantes, produites par un cerveau malade. C'est un art d'aliéné qui montre le monde tel qu'un malade (biologie épuisée ou mélangée) le voit.

Dès lors, comme l'a très bien montré Éric Michaud', il doit être écarté, non seulement comme symptôme mais également comme foyer d'infection. Produit en

\begin{abstract}
Johann Chapoutot
est professeur d'histoire

contemporaine à Sorbonne

université. Spécialiste

de l'histoire de l'Allemagne

et du nazisme, il a notamment publié Le nazisme et l'antiquité (2008), traduit en cinq langues

(Greeks, Romans, Germans.

How the Nazis usurped

Europe's Classical Past, UCP, 2016) et La loi du sang. Penser

et agir en nazi (2014), traduit

également en cinq langues

(The Law of Blood, Harvard UP, 2018).
\end{abstract}

Arno Gisinger est né en 1964 en Autriche et vit aujourd'hui

à Paris. Artiste et historien

de formation, il travaille sur

les relations entre mémoire,

histoire et représentations

visuelles. Il développe une pratique artistique singulière qui lie photographie

et historiographie. Plusieurs

de ses travaux portent sur

I'exil, la guerre et la spoliation. Il est aussi enseignantchercheur, maître de conférences au département Photographie de l'université Paris 8.

Emmanuelle Polack est docteure en histoire de l'art, elle a été responsable des archives du Musée 
des monuments français

à la Cité de l'Architecture et du Patrimoine, chercheuse associée à I'Institut national d'histoire de l'art. Elle a étudié en particulier le travail d'inventaire de Rose Valland pendant les années 1940-1945 au Jeu de Paume, et le marché de l'art sous l'Occupation.

Juliette Trey est conservatrice en chef, chargée des dessins français et italiens et adjointe au directeur du département des Arts graphiques, au musée du Louvre. Elle a auparavant été conservatrice au château de Versailles, en charge des peintures du XVIII ${ }^{\mathrm{e}}$ siècle et des pastels. Elle est membre du groupe de travail sur les recherches de provenance depuis fin 2015.

Après avoir été Professeur d'histoire de l'art et d'éducation artistique à l'Universität

Koblenz-Landau, Christoph

Zuschlag occupe, depuis avril 2018, la chaire Alfried Krupp von Bohlen und HalbachProfessur für Kunstgeschichte der Moderne und der

Gegenwart (19.-21. Jh.), avec un accent sur la recherche de provenance / I'histoire des collections, à l'institut d'Histoire de l'art de I'Universität Bonn.

Ses principaux domaines de recherche sont l'art moderne et contemporain,

I'histoire des institutions artistiques et la critique d'art, la politique artistique du national-socialisme, la recherche de provenance et I'histoire des collections. spectacle et dument encadré et commenté, il peut servir à l'édification populaire, à cette Volksaufklärung dont Joseph Goebbels est le ministre. Une fois cette tâche remplie, il peut être détruit. Il le fut en partie, certes, mais il fut plus majoritairement et plus sûrement revendu par un Reich en quête de devises.

Les nazis ont été en effet des prédateurs comme rarement l'histoire en a connus. Leur économie tout entière a reposé, dès 1933, sur la spoliation interne des citoyens allemands exclus de la cité, qu'ils aient été incarcérés ou poussés à l'exil, et sur les perspectives de prédations externes, sur les dépouilles opimes d'une guerre à venir. Du blé ukrainien au lait danois, en passant par les wagons de la SNCF ou les ampoules, les fils de cuivre et les étagères saisis dans les appartements des Juifs français privés de leurs logements, les nazis raflaient tout, afin que les Allemands ne manquent de rien et que la mauvaise idée de se révolter contre le pouvoir en place ne les reprenne pas comme aux temps honnis de 1917-1918. Dans cette économie de la rapine généralisée, les œuvres d'art revêtaient une importance particulière, en raison de leur valence esthétique et testimoniale (en ce qu'elles témoignaient du génie de la race nordique) ou de leur valeur marchande. Les premières étaient volées pour la jouissance particulière d'un Goering, dont les sicaires tentaient de prendre de vitesse les Kommandos spéciaux de Rosenberg, pour le parti (l'Einsatzstab Reichsleiter Rosenberg), d'Himmler pour la SS et l'Ahnenerbe, et d'Hitler pour ses collections personnelles et pour ce fameux musée qu'il offrirait à son peuple, à Linz. Les secondes, après avoir été clouées au pilori d'expositions infamantes, terminaient sur un marché parallèle qui devait rapporter de fraîches devises à un Reich qui en manquait tant.

Pour explorer les différents enjeux liés à une étude et à une réflexion sur l'art dégénéré et la spoliation, la revue Perspective a réuni plusieurs experts de la question, dont les compétences ressortissent à des domaines et à des spécialités différents. Emmanuelle Polack est historienne de l'art, co-éditrice des carnets de Rose Valland, elle est une spécialiste reconnue des activités de l'Einsatzstab Reichsleiter Rosenberg, l'une des multiples agences qui, comme nous venons de le voir, et en concurrence avec l'Ahnenerbe de la SS et les services personnels d'Hermann Goering, sillonnaient l'Europe à la recherche de proies patrimoniales. Christoph Zuschlag est professeur d'histoire de l'art à l'Universität Koblenz-Landau, spécialisé depuis quelques années en Provenienzforschung, en étude sur la provenance des cuvres. Depuis avril 2018, il occupe la chaire Alfried Krupp von Bohlen und Halbach-Professur für Kunstgeschichte der Moderne und der Gegenwart (19.-21. Jh.), avec un accent sur la recherche de provenance / l'histoire des collections, à l'institut d'Histoire de l'art de l'Universität Bonn. Arno Gisinger est artiste. Maître de conférences à l'université Paris 8, il travaille volontiers sur le document et la trace, archivistique ou objectale, de l'histoire. Il a consacré une partie de son travail aux objets spoliés. Juliette Trey, enfin, est conservatrice au département des Arts graphiques du musée du Louvre, en charge des dessins français et italiens du XVIII siècle. Elle fait partie du groupe de travail sur la provenance des cuvres récupérées après la Seconde Guerre mondiale (MNR).

Ces éclairages multiples, de l'histoire à la pratique de l'art, de la conservation à l'appréhension juridique des objets, nous permettent de mieux cerner une question qui peut, aujourd'hui, sembler anecdotique ou absurde - celle d'un art considéré comme dégénéré et condamné à l'invisibilité, dont le sort fut si emblématique de la vision du monde et des pratiques nazies. [Johann Chapoutot]

Johann Chapoutot. En quoi consiste l'épuration de l'art allemand? Quels sont les critères des nazis pour qualifier une cuvre de "dégénérée "?

Emmanuelle Polack. En Allemagne, les attaques contre l'art moderne furent initiées bien avant l'accession d'Adolf Hitler au pouvoir. La crise socio-économique des années 1930, le chômage croissant et la montée du nazisme catalysèrent les charges contre la vie 
culturelle initiée sous la république de Weimar, en général, et les foyers avant-gardistes, en particulier. Les propositions d'un art subjectif, dont la force créatrice rejetait les canons académiques et s'émancipait dans l'éclatement des formes, la puissance des couleurs, la liberté des thèmes abordés, I'audace des représentations, ne pouvaient qu'effrayer les tenants d'un retour au classicisme des formes. Paul Schultze-Naumburg (1869-1949), architecte allemand, futur responsable des revues d'art du IIle Reich, publiait en 1928 L'Art et la race, un mélange de thèses eugénistes et artistiques, dont le propos relevait d'une farouche opposition entre l'influence du cosmopolitisme dans l'art moderne et le " génie nordique ». Resté célèbre parmi les écrits de l'histoire de I'art allemande de cette période, Schultze-Naumburg y met en scène un montage de reproductions d'œuvres d'art issues des courants artistiques novateurs et de photographies de personnes atteintes de difformités physiques. L'auteur, par ce parallèle, frappait les esprits, tout en dénonçant ce qu'il considérait comme le résultat d'un modernisme international et d'une surreprésentation des artistes juifs, soufflant un esprit « dégénéré » sur la communauté allemande.

En ce sens, le 30 juin 1937, Adolf Ziegler (1892-1959), l'artiste-peintre préféré d'Adolf Hitler, président de la chambre des Beaux-Arts du Reich, obtint, par l'intermédiaire de Joseph Goebbels, la mission de s'emparer de toutes les œuvres d'art jugées " décadentes » des collections nationales ou privées pour les faire figurer dans une exposition intitulée Entartete Kunst, inaugurée le 19 juillet 1937 à Munich. Ainsi plusieurs milliers de peintures, sculptures, dessins et estampes furent-ils confisqués dans les musées allemands sous le III Reich. Les plus forts prélèvements ont été opérés au Folkwang-Museum d'Essen, à la Kunsthalle de Hambourg, dans les collections de la ville de Düsseldorf, au Kupferstichkabinett de Berlin, aux musées d'Erfurt, aux musées de Francfort, à la Kunsthalle de Mannheim, au Schlesisches Museum de Breslau, à la Galerie nationale de Berlin, à la Städtischen Bildergalerie de Wuppertal-Elberfeld, aux Staatliche Kunstsammlungen de Weimar, à la Staatliche Galerie de Stuttgart, au musée municipal et au Kupferstischkabinett de Dresde, au Städtischen Kunstssammlungen de Chemnitz, au musée Wallraf-Richartz de Cologne, au Landesmuseum d'Hanovre, et au Kunstgewerbemuseum de Hambourg. Entre les mois d'août et d'octobre 1937, tous les musées allemands furent épurés de leurs collections d'art moderne.

Christoph Zuschlag. La notion d'« art dégénéré » fut l'arme de propagande principale utilisée par les nazis pour mener leur combat contre l'art moderne. Elle désigne tant les nombreuses réquisitions d'œuvres d'art dans les musées allemands en 1937 que les deux expositions itinérantes présentées de 1933 à 1937 (conçue à Dresde) et de 1937 à 1941 (élaborée à Munich)². Le combat contre l'art moderne faisait partie intégrante d'une politique artistique dont le seul objectif visait à propager l'idéologie nazie et à construire des images de l'ennemi.

La première vague de réquisitions eut lieu peu de temps avant l'inauguration de l'exposition Art dégénéré sous les arcades du Hofgarten à Munich le 19 juillet 1937. Adolf Ziegler avait reçu, comme l'a mentionné Emmanuelle Polack, un décret du ministre de la Propagande, Joseph Goebbels, qui l'autorisait à « sélectionner et confisquer les œuvres - tableaux et sculptures créés à partir de 1910 - appartenant au Reich, aux régions et aux communes, et symptomatiques du déclin de l'art allemand, en vue de les présenter dans le cadre d'une exposition ». Ce fut une initiative sans précédent, d'une ampleur qui dépasse l'entendement. Ce décret représentait une atteinte grave aux droits des régions et des communes, et en particulier aux prérogatives du ministre de I'Éducation Bernhard Rust, dont dépendaient les musées. Cette réorganisation est, dès lors, révélatrice de la rivalité et de la lutte de pouvoir que se livraient les deux ministres, Goebbels et Rust, en vue d'avoir la main sur la politique artistique. Conformément 
au décret de Goebbels, Ziegler ordonna la réquisition de centaines d'œuvres d'art qu'il fit transporter à Munich pour les présenter dans le cadre de l'exposition diffamatoire. Cette première réquisition fut bientôt suivie par une seconde, bien plus conséquente. Si la première opération, réalisée à la hâte, s'était réduite à un passage en revue des collections en vue de sélectionner des pièces pour l'exposition de Munich, la seconde opération avait pour objectif la liquidation totale et systématique de l'art moderne. Au total, près de 21000 œuvres d'art furent saisies par plusieurs commissions dans 101 musées. Un tiers d'entre elles était des tableaux, des sculptures, des aquarelles et des dessins, tandis que les gravures représentaient les deux autres tiers.

Il n'existait aucun critère clair et explicite qui aurait permis de définir ce que I'on entendait par "dégénéré ». Loin de reposer sur un fondement philosophique ou artistique, ce qualificatif correspondait à une invention propagandiste confuse. L'expression diffamatoire $d^{\prime}$ « art judéo-bolchevique » forgée par les nazis et employée comme synonyme $\mathrm{d}^{\prime}$ " art dégénéré » et $\mathrm{d}^{\prime}$ « art en déclin » corrobore cette analyse. Tous ces termes étant flous et per se indéfinissables, ils pouvaient être projetés sur les productions de l'art moderne dans son intégralité, sans distinction de ses différentes facettes, qui recouvraient pourtant des courants esthétiques foncièrement différents. Parmi les œuvres réquisitionnées, on dénombrait des pièces de l'impressionnisme allemand, et plus encore de l'expressionisme allemand, mais aussi du dadaïsme et du constructivisme, ainsi que des œuvres d'artistes du Bauhaus et de l'abstraction, en passant par la Nouvelle Objectivité. Les expressionnistes, en particulier ceux du groupe d'artistes dresdois Die Brücke, furent particulièrement touchés. Leur style et leur image expressive et déformée de l'homme étaient inconciliables avec le naturalisme de l'art figuratif exigé par l'État nazi car jugé plus « proche du peuple».

Il faut bien comprendre que les commissions travaillaient à un rythme effréné. Lors de la seconde vague de réquisitions, des listes répertoriant les artistes représentés à Munich et des comptes rendus de l'exposition parus dans la presse leur servirent de points de repère, mais leur sélection fut en définitive autant subjective que contradictoire. Ainsi, elles ordonnèrent la saisie d'estampes dans un musée, alors que d'autres tirages des mêmes gravures ne furent ni réclamés ni confisqués. On peut aussi citer un exemple concret dans le domaine de la sculpture : le Portrait de l'actrice Annie Mewes d'Edwin Scharff, datant de 1917, fut saisi le 12 juillet 1937 dans les collections de peintures de l'État de Bavière, tandis que le moulage de la même sculpture en bronze de la Städtische Kunsthalle de Mannheim échappa à la réquisition.

Johann Chapoutot. Le cas du marchand d'art Hildebrand Gurlitt est en soi très instructif, notamment en ce qu'il montre que les nazis avaient besoin d'experts pour désigner les cuvres néfastes à l'esprit de la race. A-t-on connaissance de cas analogues?

Christoph Zuschlag. Je ne sais pas si je qualifierais le cas de Hildebrand Gurlitt d'« en soi très instructif ». Mais vous avez raison : un grand nombre d'experts en art, des artistes autant que des historiens de l'art et des marchands d'art, furent impliqués dans les expositions, la réquisition et surtout l' « exploitation » de " l'art dégénéré », qui débuta en 1938. À propos du rôle de Hildebrand Gurlitt : le 31 mai 1938 fut promulguée la " loi sur la confiscation des produits de l'art dégénéré ». Elle permettait la confiscation sans indemnisation des œuvres d'art réquisitionnées au profit du Reich et chargeait le ministre de la Propagande Joseph Goebbels de son application. L'objectif consistait à légaliser rétroactivement l'épuration des musées de 1937 et à créer une base légale visant $I^{\prime}$ « exploitation » systématique des œuvres confisquées. La création de la « commission d'exploitation des produits de l'art dégénéré », sous l'égide de Goebbels, marqua 
le début de cette " exploitation ». Certains membres de cette commission avaient déjà participé aux réquisitions et aux préparatifs de l'exposition Entartete Kunst ; d'autres, dont le marchand d'art Karl Haberstock, étaient novices. La commission d'exploitation travaillait en collaboration avec quatre marchands d'art affectés par le ministère de la Propagande à cette mission en raison de leur expérience internationale en matière de commerce « d'art dégénéré »: Bernhard A. Böhmer (1892-1945), originaire de Güstrow, Karl Buchholz (1901-1992), originaire de Berlin, Hildebrand Gurlitt (1895-1956), de Hamburg, ainsi que Ferdinand Möller (1882-1956), de Berlin. Officiellement, ils n'étaient autorisés qu'à faire du commerce avec l'étranger en échange de devises, mais ils revendaient tous les quatre des œuvres à l'intérieur du Reich. Les principaux acheteurs d' « art dégénéré » étaient des musées et des particuliers domiciliés

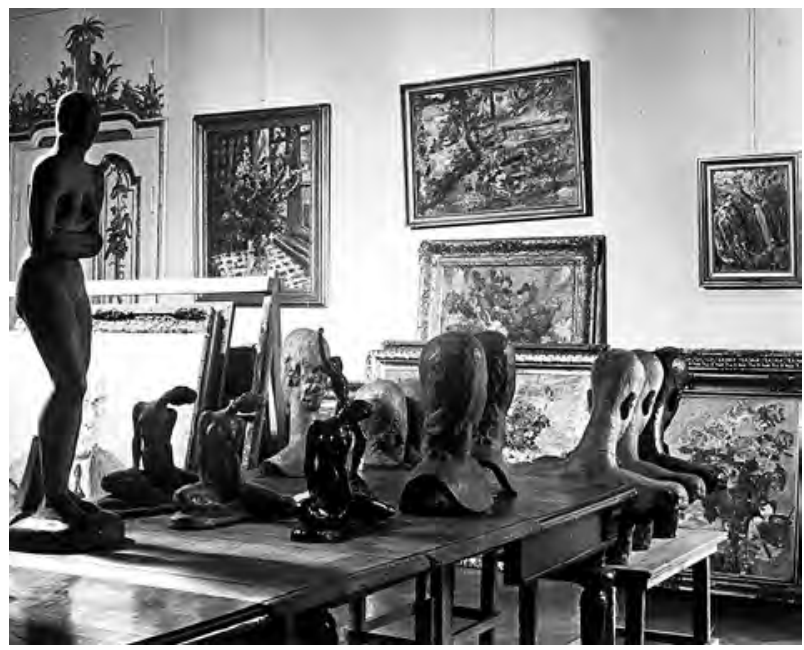
aux États-Unis, en Suisse, au Danemark, en Hollande, en Belgique, en Angleterre et en Norvège. Hildebrand Gurlitt était également impliqué dans le commerce d'œuvres d'arts spoliées.

Emmanuelle Polack. Les considérations idéologiques furent sacrifiées sur l'autel de l'effort de guerre ; les membres du parti nazi n'hésitèrent pas à mettre de côté leurs convictions idéologiques pour obtenir une manne disponible pour l'économie du Reich. À ce titre, les œuvres réprouvées parvinrent, tout d'abord, sur le marché de l'art allemand, avant qu'elles ne soient proposées sur la scène internationale. Pour ce faire, toutes traces d'appartenance aux musées furent effacées, un catalogue de six volumes fut établi par un juriste et historien de l'art nommé par le ministère de la Propagande : Rolf Hetsch (1903-1946).

Le 13 janvier 1938, Joseph Goebbels se rendit avec Adolf Hitler dans un ancien grenier à blé sur la Köpenicker Strasse, à Berlin, où s'entassaient 12890 œuvres ${ }^{3}$ confisquées des collections allemandes et dont le sort fut, à cette occasion, définitivement scellé. Comme l'a déjà mentionné Christoph Zuschlag, en mai 1938, une Kommission zur Verwertung der Produkte entarteter Kunst instituée à l'initiative de Goebbels, placée sous la présidence de I'historien de l'art Franz Hofmann (1888-?), fut en charge de la vente des produits de I' « art dégénéré ». Elle était composée de marchands d'art, tels que Karl Haberstock (1878-1956), Heinrich Hoffmann (1885-1957), Carl Meder, Robert Scholtz (1902-1981), Hans Schweitzer (1901-1980), Max Taeuber et Adolf Ziegler (1892-1959). Ce qui fut jugé sans valeur selon les critères de Franz Hofmann, soit 1004 peintures et tableaux, 3825 aquarelles, dessins, etc. fut brûlé dans la cour des pompiers à Berlin le 20 mars 1939 - un autodafé dont la symbolique participait de la politique de dévalorisation de l'art moderne. Dans le même temps, sous I'autorité de Rolf Hetsch, s'ouvrait une salle de vente au château de Schönhausen (Niederschönhausen, fig. 1), près de Berlin, pour écouler une partie du stock exploitable du dépôt de la Köpenicker Strasse. Quatre marchands d'art furent appelés en renfort pour mener à bien des négociations à l'étranger : tous partageant un goût prononcé pour les œuvres des avant-gardes allemandes, ils n'hésitèrent en aucune façon à garder par devers eux des œuvres destinées à l'importation. Ainsi, parmi les 1258 œuvres retrouvées à Munich dans la collection de Cornelius Gurlitt (1932-2014), fils de Hildebrand, près de 300 pourraient avoir transité par l'exposition Entartete Kunst de 1937 et, en amont, par le dépôt de la Köpenicker Strasse. 


\begin{abstract}
Johann Chapoutot. Parallèlement à l'épuration des musées, la spoliation artistique a consisté à priver les propriétaires légitimes de leur patrimoine. Étaient-ce les mêmes personnes qui furent chargées de faire le tri dans les collections publiques et dans les collections privées?

Qu'ont-elles saisi majoritairement? Quelle est la proportion d'œuvres détruites, revendues?
\end{abstract}

Christoph Zuschlag. Sur ce point, il est nécessaire de faire la distinction entre deux types d'œuvres d'art : comme je viens de l'expliquer, la campagne contre ce que les nazis appelaient l' « art dégénéré » concernait les œuvres d'art moderne issues des fonds des musées allemands. Les collections privées n'étaient pas touchées et il n'était d'ailleurs pas interdit de posséder des œuvres $d^{\prime}$ " art dégénéré ». Il en allait autrement de l'art spolié. L'art spolié, plus précisément l'art spolié par les nazis (NS-Kunstraub), désigne autant les œuvres d'art de toutes les époques que les biens culturels de toute sorte (dont des objets de la vie quotidienne, comme l'argent et le mobilier) qui furent « soustraits » à leur propriétaire - l'ayant-droit étant un particulier - durant la période nazie « à la suite de persécutions nazies ». Les victimes de spoliations étaient majoritairement des Juifs qui furent contraints de vendre leurs biens par le régime ou qui furent expropriés par l'État dans le cadre du processus $d^{\prime}$ « aryanisation ». Ces mesures s'inscrivent dans le contexte de la persécution des Juifs perpétrée par l'État nazi pour des motifs raciaux qui débuta peu de temps après l'arrivée de Hitler au pouvoir le 30 janvier 1933. Après la promulgation des Lois de Nuremberg au Congrès du NSDAP le 15 septembre 1935, la persécution des Juifs s'amplifia, ceux-ci furent démis de leurs droits avant d'être assassinés par millions dans les camps d'extermination durant toute la Seconde Guerre mondiale. Au cours de ce processus, l'État accéléra leur émigration jusqu'en 1941 en développant parallèlement un système perfide en vue de s'approprier le patrimoine des Juifs désireux de s'exiler. Jusqu'en 1941 il existait à Berlin une institution appelée "Office pour la Palestine » qui permettait à des groupes sionistes d'organiser, sous l'égide de l'Agence juive pour la Palestine, l'immigration de Juifs allemands vers la Palestine. En 1941 les nazis changèrent de politique et optèrent pour la "Solution finale » : on entama la construction de camps de concentration, comme Auschwitz, afin d'y déporter systématiquement les Juifs de toute l'Europe ; la Shoah débuta en 1942.

Qu'entend-on en fin de compte, concrètement, par « soustraction d'œuvres d'art à la suite de persécutions nazies » ? Arrêtons-nous un instant sur la « taxe d'émigration » pour mieux comprendre ce phénomène. Cet impôt n'était pas une invention nazie, au contraire : il fut introduit dès le 8 décembre 1931, à la fin de la république de Weimar, par le gouvernement bénéficiant d'une légitimité démocratique, en réaction à la crise économique mondiale. Les résidents désireux de quitter le territoire, détenteurs d'un patrimoine de plus de 200000 Reichsmarks, devaient céder $25 \%$ de leur fortune à I'État. Cette mesure visait à dissuader les citoyens allemands de partir à l'étranger et à enrayer la fuite des capitaux. Les nazis firent de cet impôt un instrument qui rendit possible la saisie systématique du patrimoine des Juifs sur le point d'émigrer. Mais une fois qu'ils s'étaient acquittés de cette «taxe d'émigration », les émigrants juifs ne pouvaient s'exiler avec le reste de leur patrimoine. Leurs avoirs bancaires et mobiliers étaient déposés sur des comptes bloqués qui ne pouvaient être transférés à l'étranger qu'en contrepartie d'un acompte élevé. Dans l'urgence causée par les persécutions, nombre de familles juives furent contraintes de se séparer de tous leurs biens, écoulés lors de « ventes aux enchères de biens juifs ». Les œuvres d'art et autres objets revendus de la sorte relèvent de la spoliation. Il faut aussi évoquer le « décret sur l'utilisation des biens juifs » du 3 décembre 1938 qui accéléra I' « aryanisation » des biens patrimoniaux juifs, y compris les objets d'art de toute sorte. Les collections d'art qui étaient encore 
aux mains de propriétaires juifs furent systématiquement saisies et « exploitées ». L'instance chargée de cette mission n'était pas une commission (comme c'était le cas pour la confiscation des œuvres $\mathrm{d}^{\prime}$ " art dégénéré »), mais la plupart du temps la Geheime Staatspolizei (Gestapo), très crainte par la population.

À ce stade, je peux seulement donner des chiffres tangibles concernant $I^{\prime}$ " art dégénéré ». D’après une étude statistique menée par mon collègue Andreas Hüneke du Centre de recherche sur I' « art dégénéré » de la Freie Universität de Berlin, sur les 21000 œuvres d'art saisies, $46 \%$ ont été vendues ou échangées et $33 \%$ détruites. Les $21 \%$ restants correspondaient à des invendus que la commission ne put écouler auprès des marchands d'art, des pièces de l'exposition itinérante $d^{\prime}$ " art dégénéré », des cas isolés de restitutions à des musées, etc.

Johann Chapoutot. Quelles sont les structures administratives en charge de la spoliation dans le Altreich, puis dans les territoires agrégés en 1938 ? La spoliation devient à partir de 1939 un Kunstraub. Quelles sont les agences en charge de cette spoliation? Comment la prise en charge physique des auvres est-elle assurée? Des historiens de l'art, des techniciens sont-ils impliqués? Quelles sont les modalités de transport, de stockage et de conservation? Les compagnies d'assurance allemandes sont-elles partie prenante?

Christoph Zuschlag. C'est la chambre des Beaux-Arts du Reich qui avait la charge de la réquisition et de $\mathrm{I}^{\prime}$ « exploitation » de I' « art dégénéré ». Elle était le seul organe de la chambre de la Culture du Reich à dépendre directement du ministère du Reich à l'Éducation du peuple et à la Propagande. Le ministre de la Propagande Joseph Goebbels en personne était à la tête de la « commission d'exploitation des produits d'art dégénéré » qui se réunit au total sept fois entre le 25 mai 1938 et le 11 décembre 1941.

Quant aux œuvres d'art spoliées, ce sont les différentes autorités financières et les institutions de l'État nazi ainsi que la SS et la Gestapo qui étaient impliquées dans leur réquisition et dans leur traitement. Le même régime fut appliqué à l'Autriche, annexée en mars 1938, où en l'espace de quelques jours les nazis procédèrent à l'épuration de nombreuses collections d'art, entre autres celle de Louis von Rothschild. Sa collection fut dispersée dans plusieurs musées autrichiens et la République d'Autriche resta son propriétaire jusqu'en 1999. Ce n'est qu'après la déclaration de Washington du 3 décembre 1998, qui aboutit à l'adoption d'une loi autrichienne sur la restitution des œuvres d'art confisquées par les nazis, que 224 œuvres d'art furent restituées, en février 1999, aux héritiers de Louis von Rothschild, mort en 1955. Au cours de la Seconde Guerre mondiale, dans les régions d'Europe occupées par la Wehrmacht, des organisations nazies pillèrent des collections publiques et privées, des châteaux, des archives et des bibliothèques. L'historien américain Jonathan Petropoulos estime le nombre total d'œuvres d'art spoliées à 600000 : 200000 en Allemagne et en Autriche, 100000 en Europe de I'Ouest et 300000 en Europe de l'Est. Les instances impliquées dans ces actions étaient des organisations, des institutions et des personnes qui étaient pour une part fortement mises en concurrence les unes contre les autres, parmi elles l'équipe d'intervention du Reichsleiter Rosenberg (Einsatzstab Reichsleiter Rosenberg, ou ERR), le bureau Mühlmann (Dienststelle Mühlmann), le Sonderkommando Paulsen de l'Office central de la sécurité du Reich, le bureau militaire allemand de protection de l'art de la Wehrmacht, les experts de la « Mission spéciale de Linz » (Sonderauftrag Linz) et les ambassades d'Allemagne. L'acheminement des œuvres $d^{\prime}$ " "art dégénéré » était assuré par l'entreprise de transport internationale Gustav Knauer dont le siège était situé à Berlin. Cette société avait aussi un bureau à Paris et était également impliquée dans le transport d'œuvres d'art spoliées vers l'Allemagne. 
2. Raphaël Denis, La Loi normale des erreurs, installation (cadres anciens et graphite sur bois), 2014-2018.
Johann Chapoutot. Arno Gisinger, comment l'art et les images peuvent-ils se saisir de la question des spoliations?

Parallèlement, l'épuration de l'art allemand a consisté à désigner les œuvres néfastes à l'esprit de la race. L'art d'aujourd'hui peut-il rendre compte d'une telle réalité historique?

Arno Gisinger. En effet, ces dernières années plusieurs artistes se sont saisis de la question de la spoliation. Je pense au projet Rose Valland Institute de Maria Eichhorn, présenté en 2017 lors de la dernière documenta à Cassel. La forme singulière de ce travail (une installation et une plate-forme numérique) a permis à un public intéressé par l'art contemporain de découvrir une autre approche d'une thématique fortement médiatisée depuis I'affaire Gurlitt. Par ailleurs, d'autres œuvres, comme par exemple celle de Sammy Baloji sur les pillages coloniaux, ont élargi les discussions autour de la notion de «spoliation ». Avant Maria Eichhorn d'autres artistes avaient déjà travaillé sur la tentative d'épuration de l'art allemand et européen entre 1933 et 1945. Je pense à Raphaël Denis avec La Loi normale des erreurs (2014-2015, fig. 2) ou Vernichtet (2015), à Anna Artaker avec la Rekonstruktion der Rothschild'schen Gemäldesammlung in Wien (2013) ou à Simon Starling et son travail Nachbau (2007) au Museum Folkwang à Essen. Tous ces travaux interrogent le rôle des différents protagonistes du monde de l'art pendant la Seconde Guerre mondiale : artistes, collectionneurs, galeristes, marchands d'art, musées, maisons de ventes, etc. Ces œuvres ont en commun de proposer des réflexions artistiques sur la question de l'art face aux fascismes et aux totalitarismes.

Mais il faut replacer la question de l'art dans le contexte général de la spoliation des biens juifs en Europe. Même si elles ont une très grande valeur symbolique, les œuvres d'art ne représentent qu'une infime partie de ce vol systématique

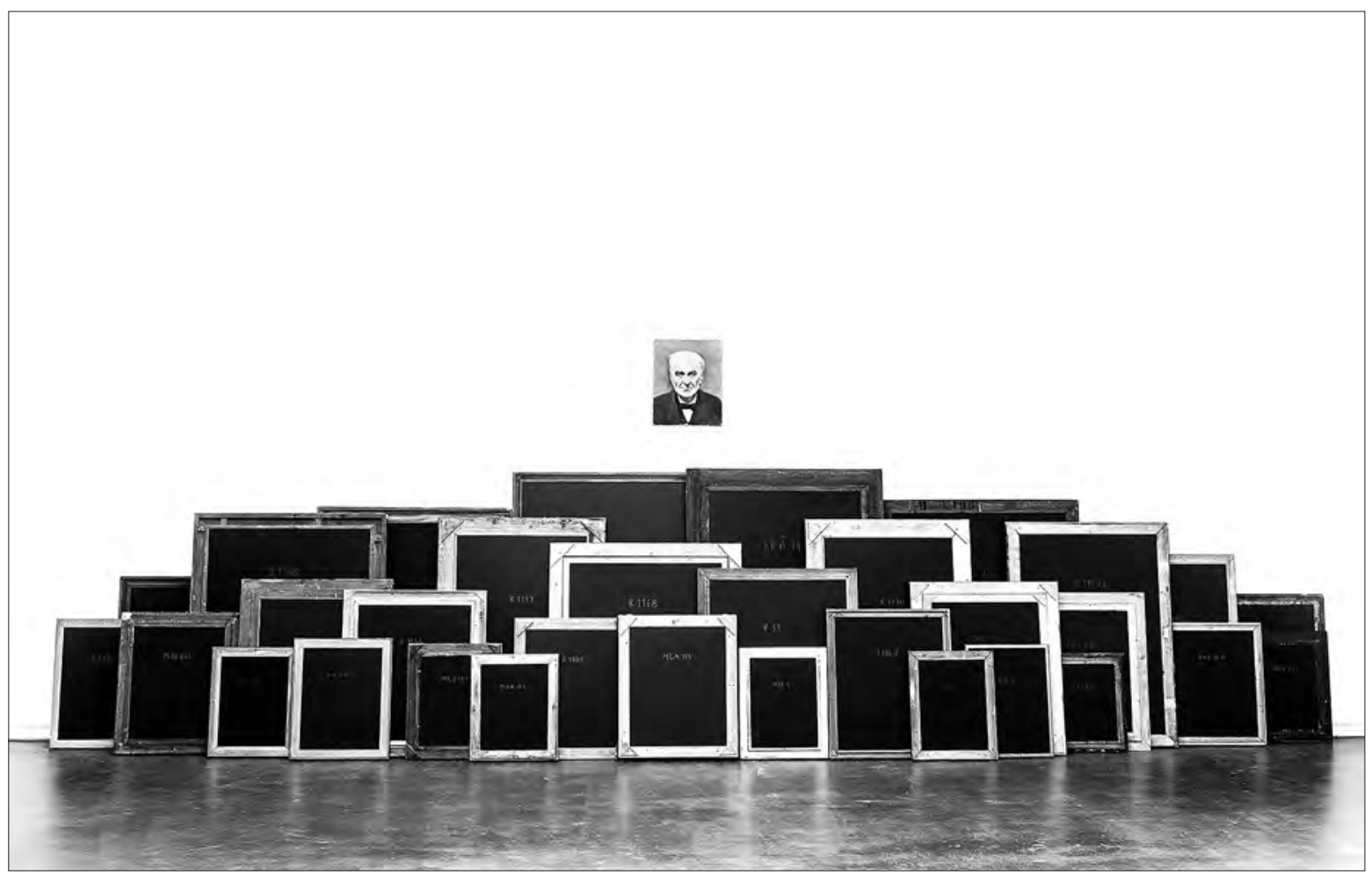


et à grande échelle. La pièce sonore No One Returns, $N^{\circ} 1$ que Laetitia Badaut Haussmann a présentée en 2010 au Palais de Tokyo fait partie des œuvres qui se mesurent à la question de la spoliation au sens large tout en proposant, en même temps, une autoréflexion précise sur I'un des lieux emblématiques de I'art à Paris. La pièce " invisible » faisait écho au passé du bâtiment, dont le sous-sol avait servi pendant la Seconde Guerre mondiale de lieu de stockage pour des centaines de pianos spoliés à des familles juives parisiennes.

L'art d'aujourd'hui peut bien sûr contribuer à une meilleure compréhension et à une visibilité majeure des phénomènes historiques. Je dirais même qu'il en a l'obligation : il s'agit tout simplement d'une question vitale de réflexion critique de l'art par l'art. Bien que le modus

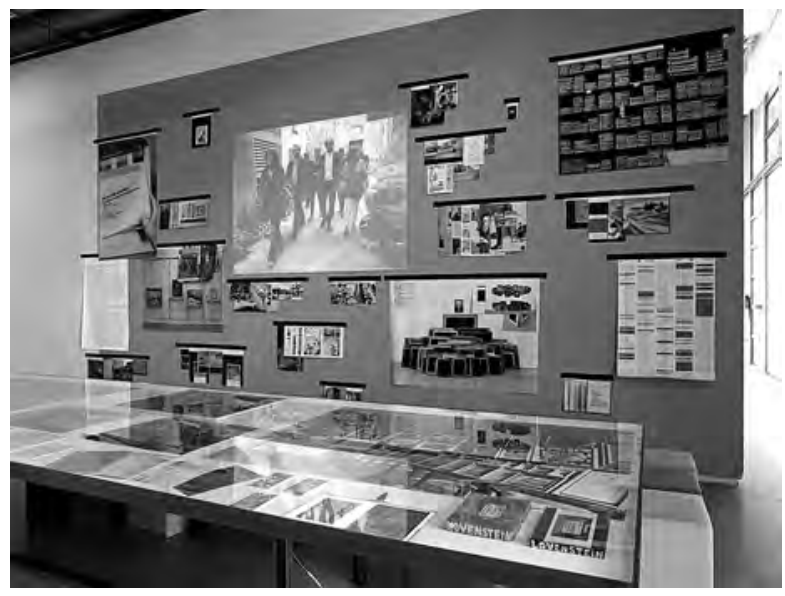
operandi des artistes soit différent de celui des historiens ou des historiens de l'art, il ne faut pas opposer les pratiques artistiques à celles des sciences humaines. D’un point de vue épistémologique il s'agit de deux façons différentes d'analyser le monde. Bien que leurs approches heuristiques et leurs formes $d^{\prime}$ expression soient distinctes, les artistes travaillent souvent avec les mêmes "sources » que les historiens, avec des documents photographiques par exemple. C'est d'ailleurs pour cela que nous avions donné le titre "Les Sources au travail » à l'université d'été 2015 de la Bibliothèque Kandinsky, qui portait sur les spoliations d'œuvres d'art par les nazis ${ }^{4}$ (fig. 3). L'idée était justement de décloisonner les approches disciplinaires et de croiser les méthodologies d'analyse pour rendre compte de la complexité et de l'actualité des enjeux d'un tel phénomène.

Johann Chapoutot. De quelles manières la photographie en particulier permet-elle d'interroger l'histoire et comment une œuvre peut-elle rendre compte notamment de l'indissociabilité de l'histoire et de la mémoire, de la mémoire et de l'oubli ?

Arno Gisinger. Les inventions de l'histoire moderne et de la photographie s'opèrent au même moment et sont consubstantielles. Selon Siegfried Kracauer, il y a une analogie fondamentale entre historiographie et photographie. Pour lui, la photographie aide à penser I'histoire, et inversement. En effet, I'image photographique a cette formidable capacité de « figurer » un objet tout en faisant sentir son absence physique. Elle reste ancrée dans le réel par l'acte de la prise de vue, mais elle transforme l'objet en une représentation visuelle. Une source qui devient preuve mais qui nécessite interprétation.

Nous connaissons également le rôle primordial de la photographie pour la constitution de I'histoire de l'art en tant que discipline. Je pense à Aby Warburg, à Walter Benjamin et bien sûr à la célèbre phrase d'André Malraux dans son Musée imaginaire de 1947 : «L'histoire de l'art depuis cent ans, depuis qu'elle échappe aux spécialistes, est l'histoire de ce qui est photographiable. » Face à un phénomène comme celui de la spoliation d'œuvres, cette phrase résonne doublement, car les documents photographiques tiennent une position centrale autant dans le travail des spoliateurs que dans le travail des historiens. Du point de vue de l'intention de leurs auteurs, on peut dire que les images photographiques se retournent souvent contre elles-mêmes.

Deux exemples peuvent illustrer cette inversion de l'usage des documents photographiques. Le premier concerne deux images prises au début des années 1940 par le service photographique allemand alors installé au Jeu de Paume, à l'époque lieu 

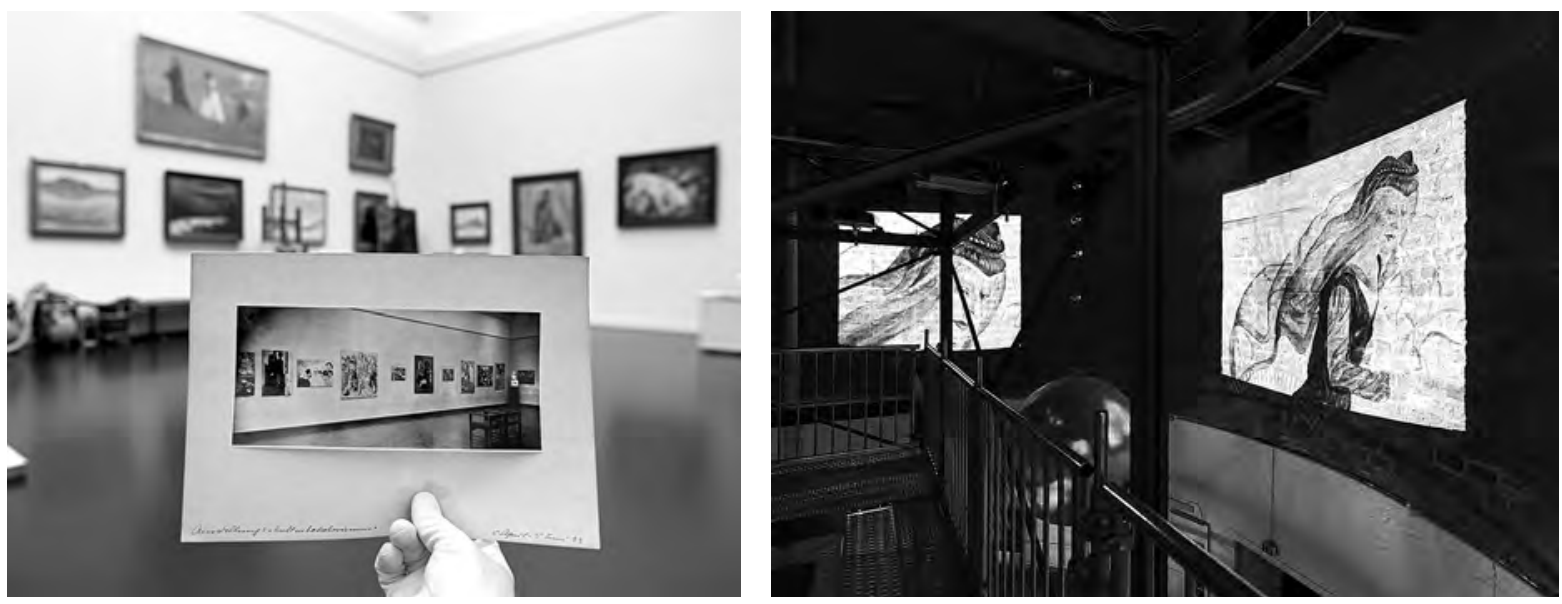

4. Arno Gisinger, Vérification, 2017, Kunsthalle Mannheim. 5. Arno Gisinger, Bilderstreit, 2017, projection dans I'ancien château d'eau de Mannheim. de stockage et de transit des œuvres volées en France par les nazis. Dans une pièce à I'accès restreint que Rose Valland avait appelé la " salle des martyrs » étaient stockées environ trois cents œuvres spoliées de tous les grands noms de l'avant-garde européenne de l'époque. Les photographies, initialement prises par les spoliateurs comme de simples photographies d'inventaire, ont servi des années plus tard aux chercheurs pour identifier les tableaux volés. La qualité des négatifs - deux prises de vue à la chambre $20 \times 25$ qui forment un panorama de la salle - ont permis de retrouver une grande partie des œuvres spoliées. Le deuxième exemple concerne un travail que j'ai eu l'occasion de réaliser sur les collections de la Kunsthalle Mannheim, en Allemagne. En 1933, le musée avait organisé, sous le titre Kulturbolschewistische Bilder, une exposition de dénonciation de sa propre collection d'avant-garde. La même institution avait été "victime », en 1937, de la confiscation d'environ six cents œuvres pour la célèbre exposition Entartete Kunst. Les clichés pris par le photographe du musée à ces occasions ont permis de reconstituer - et de distinguer - les deux phénomènes (fig. 4). Dans le cas des confiscations de 1937, les photographies restent parfois les seuls témoins de tableaux disparus, comme par exemple La Veuve d'Otto Dix de 1925 (fig. 5).

Johann Chapoutot. Quel est, outre les collections privées de Goering et le projet de musée de Hitler à Linz, le sort des œuvres spoliées?

Christoph Zuschlag. Le droit de préemption du 18 juin 1938, qui ne s'appliquait à l'origine qu'aux œuvres d'art spoliées en Autriche et en particulier à celles issues de la collection Rothschild, mais qui fut progressivement étendue à tout le Reich allemand et aux régions occupées, en particulier à l'Est, conférait à Hitler le droit de disposer personnellement de l'intégralité de l'art spolié (fig. 6). Les œuvres d'art dont il ne voulait pas s'accaparer pour son projet de musée à Linz devaient être réparties entre les autres musées du Reich après la guerre. Les biens culturels et artistiques spoliés furent entreposés dans des dépôts à l'abri des bombardements, dont deux d'entre eux se trouvaient au Führerbau à Munich et dans l'abbaye désacralisée des Bénédictins de Kremsmünster. En mai 1944, les fonds de Munich et Kremsmünster furent mis à l'abri dans la mine de sel d'Altaussee.

Johann Chapoutot. Quelle est l'attitude des Alliés occidentaux et soviétiques face aux collections d'œuvres spoliées découvertes en 1945 ? 

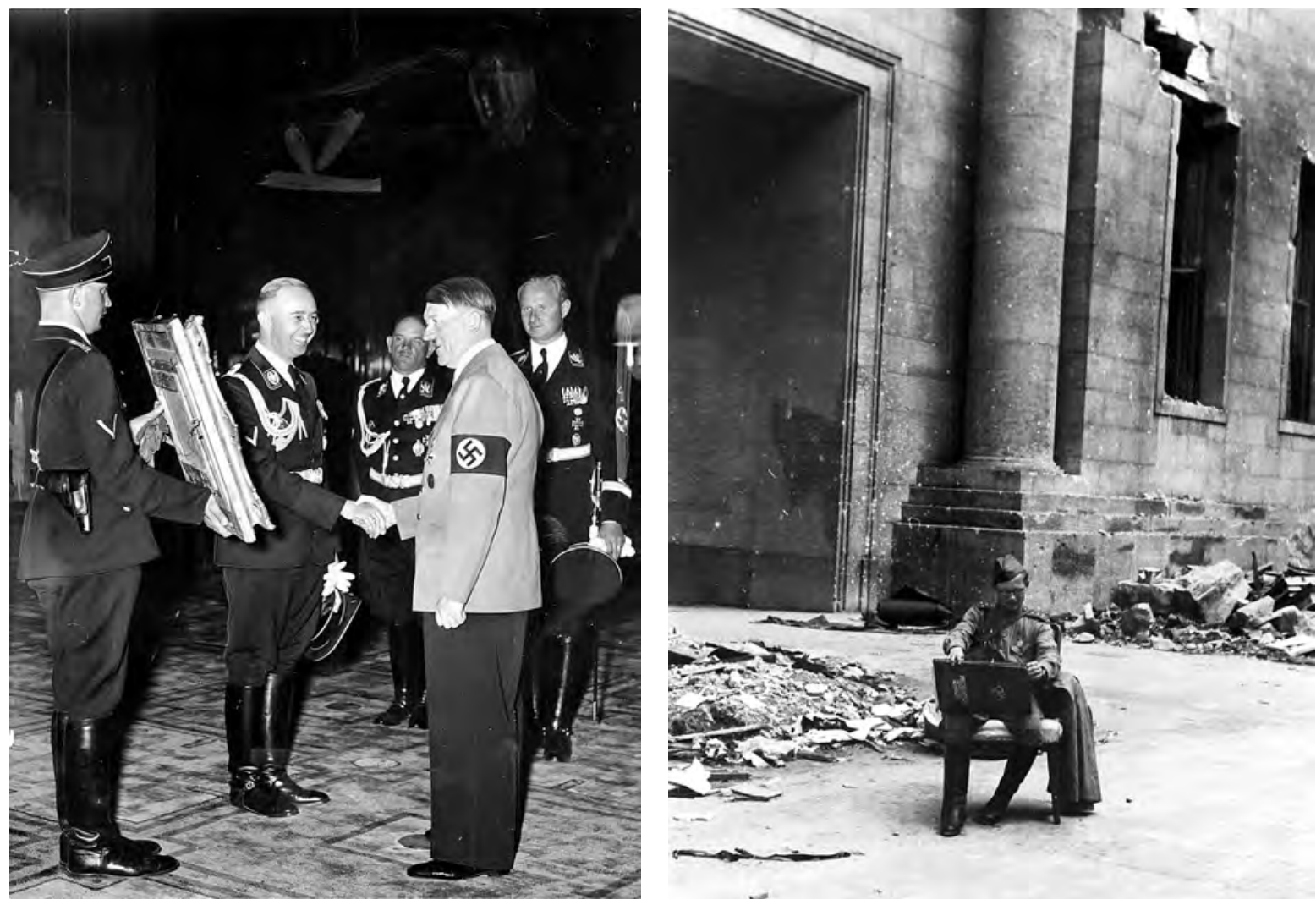

Emmanuelle Polack. À Washington, la communauté culturelle américaine fit pression sur le président Franklin Delano Roosevelt, dont on savait par ailleurs qu'il était un grand amateur d'art, pour l'établissement d'une commission gouvernementale chargée de la protection des monuments et des œuvres d'art mis en danger par les ravages de la guerre qui sévissait en Europe. S'il s'agissait certes dans un premier temps de les protéger, la question de leur récupération et de leur restitution fut très vite soulevée. L'American Commission for the Protection and Salvage of Artistic and Historic Monuments in War Areas, plus connue sous le nom de Commission Roberts, fut ainsi créée le 23 juin 1943. L'une de ses réalisations les plus importantes fut la mise en place du programme «Monuments, Fine Arts and Archives » (MFAA) et de ses officiers « BeauxArts » : les Monuments Men. Désormais rendus célèbres par le film éponyme de George Clooney, leur groupe, constitué d'environ trois cents quarante-cinq femmes et hommes, originaires de treize nations, se déploya dans le sillage du débarquement allié en Normandie sur les lignes de front. Parvenus en Allemagne en avril 1945, soit un mois avant la signature de l'armistice, les Monuments Men découvrirent peu à peu plus de deux mille caches dans des lieux aussi divers que des châteaux, des mines de sel, des caves où était dissimulé un nombre incalculable d'œuvres d'art. Celles-ci, prises en charge sans coup férir, furent rassemblées dans des Collecting Points, à partir desquels il appartenait aux divers comités nationaux de mener des recherches approfondies sur leurs provenances.

Si les Alliés occidentaux souhaitèrent rendre assez vite les œuvres confisquées principalement aux familles juives, les Soviétiques leur opposèrent immédiatement une toute autre conception : ils estimèrent que les préjudices occasionnés par l'armée
6. Heinrich Himmler offre une œuvre d'art à Adolf Hitler le 19 avril 1939 pour son $50^{\mathrm{e}}$ anniversaire, Bundesarchiv.

7. Officier soviétique dans la cour de la Chancellerie, Berlin, 1945, Paris, mémorial de la Shoah. 
allemande sur leur territoire, endommageant irrémédiablement leur patrimoine culturel, méritaient, à tout le moins, des réparations en nature. Dans la zone d'occupation soviétique en Allemagne et dans les pays d'Europe de l'Est libérés par l'Armée rouge (fig. 7), les restitutions ne furent pas à l'ordre du jour dans l'immédiat après-guerre. Il est intéressant de noter que cette assertion est toujours valable aujourd'hui. Les « Brigades des trophées » de l'Armée rouge, émanation d'un décret promulgué le 21 février 1945 par Joseph Staline en personne, élaborèrent en ce sens un plan de récupération compensatoire. Loin d'être préoccupées par la seule protection des bâtiments, à l'instar des Monuments Men, les Brigades mirent plutôt l'accent sur les précautions dans le maniement des œuvres qu'elles chargèrent dans les camions à destination de la mère patrie. La récupération des œuvres de premier ordre participa de la glorification de l'armée victorieuse en allant sans tarder orner les cimaises des plus grands musées russes.

Christoph Zuschlag. Le 8 mai 1945, jour de capitulation de la Wehrmacht, marqua la fin de la Seconde Guerre mondiale déclenchée par I'Allemagne. Dès le 13 mai, des officiers américains en charge de la protection des biens culturels s'emparèrent de la mine de sel d'Altaussee. À partir du 17 juin, les œuvres d'art furent transférées à Munich, au Central Collecting Point qui se trouvait au Führerbau et dans le bâtiment de l'administration du NSDAP. Le Central Collecting Point de Munich était le principal point de collecte d'art spolié par les nazis ; des œuvres d'art en provenance des trois zones d'occupation occidentales y étaient acheminées, référencées. On cherchait à identifier leur provenance et, selon un processus qui s'échelonna jusqu'en 1951, à les restituer, dans la mesure du possible, à leurs propriétaires. Les Soviétiques transférèrent de nombreux biens culturels de leur zone d'occupation vers I'Union Soviétique, ces trophées de guerre étant destinés à être exposés dans un musée des butins de guerre imaginé par Staline. Une grande partie d'entre eux fut restituée à la RDA et à d'autres États membres du Pacte de Varsovie entre 1955 et 1958. Les quelque 200000 objets d'art qui provenaient initialement d'Allemagne et qui se trouvent aujourd'hui encore en Russie, ainsi que près de deux millions de livres et d'importants fonds d'archives font l'objet de batailles juridiques entre la Russie et l'Allemagne depuis des années. Le célèbre "Trésor de Priam », découvert et sauvé par Heinrich Schliemann à Troie au XIXe siècle, fait partie de ces litiges. Il est exposé dans la collection permanente du musée Pouchkine de Moscou depuis 1996.

Laissons à présent de côté l'art spolié par les nazis et le " butin artistique » (Beutekunst) pour en revenir à l'« art dégénéré ». Pour ce dernier cas, les revendications de restitutions $n^{\prime}$ ont aucun fondement juridique parce que la « loi sur la confiscation des produits de l'art dégénéré » du 31 mai 1938 que j'évoquais plus haut ne fut pas abrogée par le Conseil de contrôle allié ni par le pouvoir législatif ouest-allemand après la guerre. Officiellement, elle resta en vigueur jusqu'en 1968 et ne devint caduque qu'après sa non-publication au Journal officiel de la République fédérale d'Allemagne. Par conséquent, d'un point de vue purement juridique, les réquisitions $\mathrm{d}^{\prime}$ " art dégénéré », comme l'a établi le juriste Carl-Heinz Heuer, " ont été perpétrées légalement, si bien que les acquéreurs ultérieurs devinrent aussi les propriétaires légaux de ces œuvres. [...] Aussi moralement condamnable que fût la persécution de I'"art dégénéré", aucune restitution, d'un point de vue purement juridique, ne peut être exigée ${ }^{5}$. »Ce que l'on sait moins, c'est que l'administration militaire de la zone d'occupation soviétique abrogea unilatéralement, par le décret du 8 octobre 1945, la loi nazie de réquisitions des biens culturels. Sur la base de ce décret, l'Office d'éducation du peuple saisit auprès de la municipalité du Grand Berlin des œuvres d' « art dégénéré », dont les marchands Bernhard A. Böhmer et Ferdinand Möller avaient fait I'acquisition, et les rendit aux musées de la zone d'occupation soviétique en 1949 
et au début des années 1950. Hormis les biens artistiques conservés par Böhmer et Möller, je n'ai pas connaissance d'autres applications de ce décret de l'administration militaire soviétique en Allemagne.

Johann Chapoutot. Quelle est la politique des institutions publiques allemandes et françaises face à cet héritage illégitime? Peut-on dégager des phases de prise de conscience et d'action?

Emmanuelle Polack. Au vu de mes récents travaux, je distingue quatre temps forts dans la politique des institutions françaises face aux nécessaires restitutions des biens juifs, oscillant régulièrement entre volontarisme $d^{\prime}$ une part et volonté de communiquer d'autre part. Lors de l'immédiat après-guerre, une première phase active consista dans la récupération et la restitution des biens culturels enlevés par l'ennemi ou sous son contrôle au cours de l'Occupation du territoire français, établissant en septembre 1949 le statut des $\mathrm{MNR}^{6}$. Une longue amnésie s'ensuivit, qui se prolongea jusqu'au milieu des années 1990 où un tournant historiographique s'opérait dans un climat propice aux questionnements du rôle du gouvernement de Vichy. Vers la fin des années 1990, une nouvelle phase volontariste, émanation de la mission confiée au grand résistant Jean Mattéoli (1922-2008), correspondait à la définition du corpus des MNR et à sa présentation au public. Or c'est véritablement en mars 2013 qu'Aurélie Filippetti, ministre de la Culture et de la Communication, initia une démarche " proactive » des musées de France, laissant augurer des marges de progrès significatives dans l'engagement de l'État français en faveur des restitutions d'œuvres spoliées suivant les principes de la déclaration de Washington de 19987. C'est aussi en ce sens qu'Audrey Azoulay, ministre de la Culture et de la Communication, réaffirmait, dès février 2016, I'enjeu éthique des restitutions et confiait à David Zivie, conseiller chargé du patrimoine et de l'architecture, une mission sur le traitement des œuvres et des biens culturels ayant fait l'objet de spoliation pendant la Seconde Guerre mondiale. Le rapport dressait un état des lieux de la gestion des biens culturels spoliés présents dans les institutions culturelles. Il fut remis à Françoise Nyssen, ministre de la Culture, en mars 2018, pour mettre en place rapidement des solutions déontologiques et nécessaires.

Christoph Zuschlag. L'historien Constantin Goschler qui enseigne à Bochum distingue deux phases de restitution des biens juifs dans l'histoire ouest-allemande d'après-guerre. Il a écrit en 2008 : «Une première phase commença peu de temps après 1945 et se poursuivit jusque dans les années 1960. [...] Elle concernait surtout les maisons, les terrains et les entreprises, en un mot tous les biens patrimoniaux qui étaient passés aux mains d'Allemands non-juifs au cours du processus d' "aryanisation". [...] Une seconde phase débuta en 1990 lors de l'unification des deux États allemands et perdure jusqu'à aujourd'hui. Si les premières mesures visaient à compenser l'absence de politique de restitution en Allemagne de l'Est en y appliquant les principes des lois de restitutions ouest-allemandes, de nouvelles revendications relatives à la restitution d'œuvres d'art aux héritiers des anciens propriétaires juifs se firent entendre plus récemment ${ }^{8}$. »

La déclaration de Washington du 3 décembre 1998, par laquelle la communauté internationale adopta des principes concernant la restitution du patrimoine confisqué à l'époque nazie, a permis une avancée conséquente. Les quarante-quatre États signataires, dont la République fédérale d'Allemagne, se sont engagés à recenser dans les musées, les bibliothèques et les archives les biens culturels « soustraits à la suite de persécutions nazies ", autrement dit volés, à en identifier les propriétaires légaux ou ayants-droit et à parvenir avec eux à une solution juste et équitable. Une restitution 
des biens peut être l'une des solutions envisagées. Cette déclaration correspond à un engagement moral librement consenti et n'a pas de caractère juridique contraignant; elle ne s'applique, de surcroit, qu'aux institutions publiques à l'exclusion des collections privées et du commerce d'objets d'art. En décembre 1999, l'État fédéral allemand, les Länder et les confédérations communales ont signé une déclaration commune concernant les institutions publiques en Allemagne, dans laquelle ils se sont engagés à recenser et restituer les « biens culturels soustraits à la suite de persécutions nazies, en particulier ceux dont les propriétaires sont juifs ». Fondé en 2008 et rattaché à I'Institut de recherche muséale des Staatliche Museen zu Berlin - Stiftung Preußischer Kulturbesitz, le Centre de recherche sur la provenance des œuvres d'art et biens culturels était chargé d'allouer des subventions publiques qui visent à faciliter les investigations que les musées, les bibliothèques et les archives doivent mener, à sa demande, dans leurs fonds, afin d'identifier des œuvres d'art susceptibles d'avoir été spoliées. Depuis 2015, c'est le Centre allemand des biens culturels spoliés, situé à Magdebourg, également responsable de la banque de données en ligne Lost Art, qui est le nouveau coordinateur de cette tâche.

Force est de constater que les recherches intensives et systématiques sur la provenance des biens culturels qui sont pratiquées en Allemagne depuis de nombreuses années ont permis d'identifier de nombreuses œuvres d'art spoliées et de procéder à des restitutions ou de parvenir à des solutions équitables et justes conformes à la déclaration de Washington. Mais il reste encore beaucoup à faire ! Il sera à I'avenir essentiel de développer la recherche sur la provenance des biens culturels dans les cursus universitaires tout comme dans la formation continue. Depuis peu, on répertorie trois postes de professeurs d'université non-titulaires (Juniorprofessur, ou Tenure Track) dans cette discipline en Allemagne : à Hambourg, à Bonn et à Munich. Par ailleurs, la fondation Alfried Krupp von Bohlen und Halbach a créé deux chaires, I'une en histoire de l'art, spécialisée dans la recherche sur la provenance des œuvres d'art et biens culturels, et l'autre en droit, spécialisée dans la juridiction de la protection du patrimoine culturel et artistique. L'un des objectifs est l'ouverture d'un Master croisant ces deux disciplines.

Juliette Trey. L'expression $d^{\prime}$ « héritage illégitime » pour désigner les œuvres issues de la récupération artistique ne me semble pas adaptée dans la mesure où ces œuvres ne font pas partie des collections des musées publics français; I'État en est seulement le gardien dans le cadre d'un " service public de la conservation et de la restitution », comme l'a récemment souligné le Conseil d'État (30 juillet 2014, arrêt d'assemblée). Il est en effet essentiel de rappeler que les œuvres issues de la récupération artistique, c'est-à-dire envoyées d'Allemagne en France à l'issue de la Seconde Guerre mondiale parce que l'on soupçonnait qu'elles provenaient de France et en étaient arrivées pendant la période de conflit, sont placées sous la responsabilité juridique du ministère de l'Europe et des Affaires Étrangères qui en confie uniquement la gestion aux musées de France avant que leur situation juridique ne soit clarifiée. Les œuvres issues de la récupération artistique ne sont pas inscrites sur les inventaires des musées et ne doivent pas subir d'intervention de restauration (pour conserver tous les indices que leur état matériel pourrait donner sur leur provenance, notamment les marques ou les étiquettes). Nous devons les exposer autant que possible pour les faire connaître, même si elles ne peuvent pas être prêtées hors de France. Parmi ces œuvres, celles dont on peut établir qu'elles ont été spoliées ont donc vocation à retrouver leur légitime propriétaire. En effet, une partie de ces œuvres ont été spoliées (directement ou indirectement) à leurs propriétaires juifs tandis que d'autres ont été vendues sans pressions et acquises de manière légitime sur le marché de l'art. Une part importante 
du travail de recherche de provenance consiste à distinguer, parmi les œuvres récupérées en Allemagne après la Seconde Guerre mondiale, celles qui ont été spoliées de celles qui ne l'ont pas été.

Si l'on parle d' « héritage » c'est donc plutôt au sens des missions de conservation, de gestion mais également de recherche de provenance qui incombent aujourd'hui encore aux musées qui conservent ces œuvres.

À la lumière de ces éléments, il est vrai que les restitutions de l'immédiat aprèsguerre ont d'abord été mises en œuvre principalement pour répondre aux demandes exprimées par les familles spoliées. Après cette première vague de restitutions, les recherches de provenance ont été poursuivies, notamment par Rose Valland. Emmanuelle Polack a rappelé à juste titre l'importance des années 1990 qui ont ouvert une nouvelle ère pour ces questions de restitution, avec notamment une exposition de ces œuvres en 1997 et la mise en ligne du répertoire des œuvres issues de la récupération, baptisé base Rose Valland ${ }^{9}$. On ne peut que regretter que les moyens alors mis en œuvre n'aient pas été maintenus. Notons également que la procédure de restitution des œuvres issues de la récupération artistique reposait sur un système de réclamation qui devait nécessairement émaner des propriétaires des biens spoliés ou de leurs ayants-droit. Il a donc fallu attendre la mise en place d'un nouveau groupe de travail en 2013, à l'initiative du ministère de la Culture, groupe de travail élargi fin 2015, dirigé et coordonné par France Legueltel, magistrate honoraire, rapporteur auprès de la Commission $\mathrm{d}^{\prime}$ indemnisation des victimes de spoliation ${ }^{10}$ (CIVS), pour que le travail de recherche de provenance soit davantage partagé entre les institutions et les musées dépositaires d'œuvres issues de la récupération artistique, dans le cadre d'une démarche volontaire $d^{\prime}$ identification des propriétaires et de leurs ayants-droit.

Johann Chapoutot. Juliette Trey, peut-on décrire les différents moyens dont un musée français, et ses conservateurs, disposent pour traiter de ces questions (de la recherche à la restitution)?

Qui est à l'initiative d'une telle recherche? Et comment procède-t-on pour retracer l'histoire d'une cuvre, écrire l'histoire de sa provenance? Pourriez-vous décrire votre méthode de travail ? En quoi le cas des œuvres spoliées est-il spécifique du point de vue des éventuels interlocuteurs sollicités, des fonds concernés ou des méthodes engagées?

Juliette Trey. Les conservateurs des musées français participent aux recherches de provenance, en lien avec les membres du groupe de travail sur les provenances des œuvres récupérées après la Seconde Guerre mondiale et plus spécifiquement avec le service des Musées de France. Le processus de restitution en lui-même est géré en revanche directement par le ministère de l'Europe et des Affaires Étrangères et le service des Musées de France. Les moyens dont nous disposons pour ces recherches de provenance sont les outils classiques : catalogues de vente ${ }^{11}$, catalogues $d^{\prime}$ exposition, catalogues raisonnés ou encore répertoire des marques de collection pour les dessins ${ }^{12}$. Nous consultons également des archives, notamment judiciaires pour les procès-verbaux des ventes publiques ou des inventaires après-décès, notariales pour les testaments. Les archives de marchands peuvent également être utiles. Enfin nous travaillons avec les fonds d'archives de la récupération artistique, conservés au ministère de l'Europe et des Affaires Étrangères, dans lesquels on trouve notamment les listes d'œuvres spoliées et réclamées par leur propriétaire ou leurs ayants-droits après la guerre.

L'initiative de la recherche peut avoir de multiples origines : elle peut répondre à la demande d'un ayant-droit ou bien être prise par un conservateur, par exemple, ou au SMF. Chaque recherche est différente. À titre d'exemple, le groupe de travail sur 


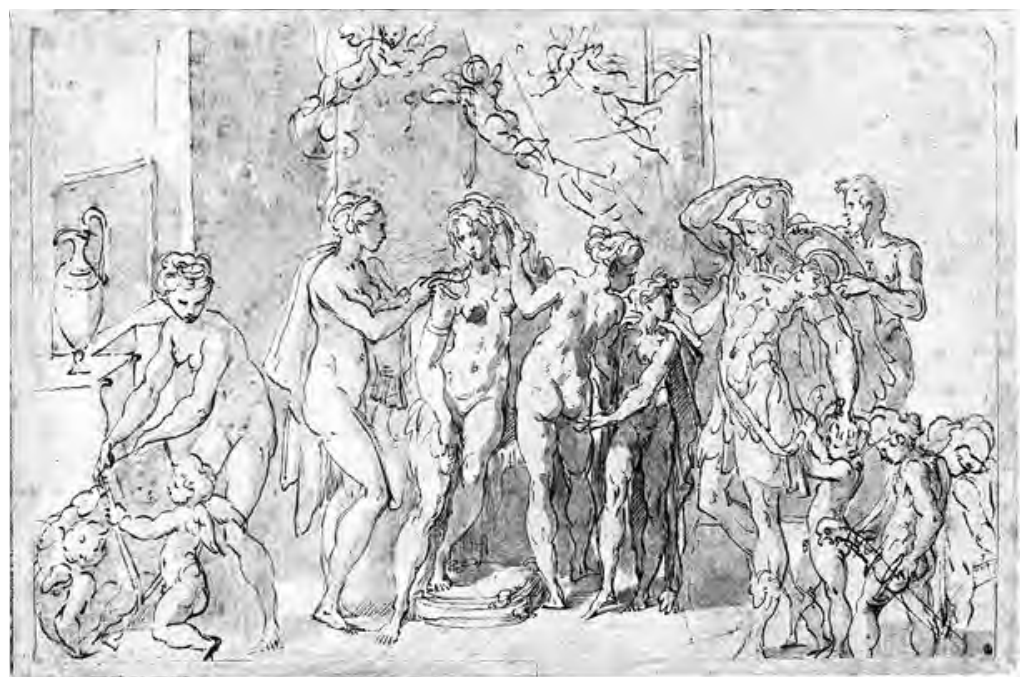

les provenances d'œuvres récupérées après la Seconde Guerre mondiale a recherché en 2016, de manière systématique, les passages en vente des œuvres issues de la récupération artistique. Ce travail a permis de préciser plusieurs historiques, grâce au dépouillement des procès-verbaux des ventes qui donnent le nom des vendeurs et des acheteurs, et même de procéder déjà à des restitutions (fig. 8). Mais une recherche peut aussi avoir comme point de départ une marque de collection ou un numéro au dos d'une œuvre.

La spécificité de ces recherches de provenance tient à la complexité de la période de la Seconde Guerre mondiale. L'histoire douloureuse des

8. Francesco Mazzuola (attr.), Les Noces d'Alexandre et Roxane, plume, encre brune et lavis brun, Paris, musée du Louvre, Fonds des dessins provenant de la Commission de récupération, inv. REC 68, restitué aux ayants-droit de son propriétaire le 13 mars 2017. spoliations nous oblige aussi à être très prudents pour éviter les erreurs et à nous assurer d'avoir un historique complet pour restituer à la bonne personne. Elle nous conduit à travailler avec les dossiers de réclamation ou d'indemnisation dressés après la guerre par les propriétaires ou leurs ayants-droit, conservés aux archives du ministère de l'Europe et des Affaires Étrangères ou encore avec des archives privées comme des testaments. Par ailleurs, il faut savoir qu'une partie des archives que nous utilisons, en raison de leur caractère privé, est soumise à une interdiction de consultation pour une durée de 75 ans par le code du patrimoine. Depuis 2018, les archives produites en 1942 sont donc librement consultables mais pas celles produites en 1943, accessibles seulement sous dérogation.

Johann Chapoutot. Comment s'articulent la politique des musées ou du musée et vos recherches dans cette perspective, et quelles sont les parts respectives, dans votre travail sur la provenance des cuvres, d'intérêt et de bon vouloir individuel d'une part, et d'application d'axes de travail explicitement définis par le musée d'autre part?

Comment concilier, lorsque l'on exerce le métier de conservatrice d'une collection publique française, le principe d'inaliénabilité avec les exigences éthiques et les obligations de la profession?

Juliette Trey. Les recherches de provenance sur les œuvres issues de la récupération artistique sont menées, au musée du Louvre, dans chaque département, par au moins un conservateur référent et membre du groupe de travail sur les provenances d'œuvres récupérées après la Seconde Guerre mondiale. Ces recherches sont soutenues et encouragées par le musée qui les coordonne et engage des moyens nécessaires pour les mettre en œuvre. Ainsi une campagne systématique de photographie des versos, des revers et des marques des œuvres issues de la récupération artistique est menée depuis quelques années. Ces photographies enrichissent la documentation numérique disponible sur la base Rose Valland et permettent d'étudier plus facilement les indices matériels relatifs à la provenance de ces œuvres.

Le principe d'inaliénabilité ne s'applique pas aux œuvres issues de la récupération artistique qui ne font pas partie des collections des musées, ceux-ci en ayant seulement la gestion. La question de l'inaliénabilité et des exigences éthiques des 
conservateurs du patrimoine se pose donc plutôt à l'occasion des acquisitions. Nous sommes ainsi très vigilants en ce qui concerne la provenance des œuvres acquises pour le musée et nous cherchons à nous assurer que ces œuvres n'ont pas fait l'objet d'une spoliation pendant la Seconde Guerre mondiale. La question plus vaste et délicate de l'examen de la provenance de l'ensemble des œuvres entrées au Louvre après 1933 n’a, à ma connaissance, pas été posée et mériterait sans doute de l'être.

Johann Chapoutot. Pourriez-vous nous en dire plus : comment la politique des musées français, et du musée du Louvre en particulier, face à ces questions, en termes de communication et d'information des publics, se matérialise-t-elle? La provenance des œuvres est-elle mentionnée sur les cartels dans les salles d'exposition ou cette mention est-elle discutée, en projet ? Les recherches sur la provenance sont-elles uniquement documentées dans les dossiers d'œuvres, disponibles à la documentation du musée ? Enfin, le musée du Louvre déploiet-il une stratégie de communication et/ou d'information spécifique des publics à cet égard?

Juliette Trey. Le musée du Louvre travaille en lien étroit avec le SMF qui gère et met à jour depuis 1997 la base Rose Valland, répertoire en ligne des œuvres issues de la récupération artistique qui présente l'état actuel des connaissances sur l'historique de ces œuvres et leurs photographies (y compris les photos des versos et des marques). Le Louvre cherche aussi à faire mieux connaître ces œuvres au public, notamment en les exposant autant que possible au sein de ses salles. Une partie des œuvres issues de la récupération artistique sont ainsi présentées au sein du parcours permanent, ou bien prêtées à l'occasion d'expositions en France et, suivant une instruction du ministère de la Culture datant du 16 octobre 2016, toujours avec une mention indiquant qu'elles sont issues de la récupération artistique : "CEuvre récupérée à la fin de la Seconde Guerre mondiale : en attente de sa restitution à ses légitimes propriétaires ». Cette mention, qui laisse entendre à tort que toutes les œuvres issues de la récupération artistique auraient été spoliées, devrait faire l'objet d'un réexamen.

Depuis la fin de l'année 2017 le Louvre présente un ensemble de tableaux issus de la récupération artistique dans deux salles qui leur sont spécialement dédiées. L'ouverture d'autres salles pour ces tableaux dits MNR est à l'étude. Des dessins et des pastels issus de la récupération artistique seront aussi exposés dans un lieu spécifique, par roulement pour des questions de conservation préventive, à partir d'octobre 2018. Le département des Sculptures prépare également un espace dédié de présentation pour la même date. Ces nouveaux espaces témoignent de la volonté du musée du Louvre de faire connaître ces œuvres le plus largement possible.

Enfin, les dossiers d'œuvres conservent les éléments relatifs à la provenance de ces œuvres et sont bien entendu librement consultables par les visiteurs dans les services de documentation des différents départements.

Johann Chapoutot. Arno Gisinger, quelle place l'archive a-t-elle dans votre travail?

Arno Gisinger. L'archive, ou plutôt les archives jouent un très grand rôle dans mon travail d'artiste, non pas comme une forme symbolique mais comme un lieu politique, un lieu de pouvoir au sens foucaldien du terme. Puisque la spoliation a été une opération planifiée et organisée de façon pseudo-légale, elle a engendré bon nombre de documents écrits et visuels. Dans deux de mes projets portant sur la spoliation, l'apport 
des archives a été primordial. Invent arisiert (2000) est un inventaire photographique des biens de Juifs autrichiens spoliés, conservés par le Hofmobiliendepot (Mobilier national) de Vienne. Un inventaire détaillé figurant dans les archives de l'institution m'a permis, en collaboration avec l'historien Herbert Posch, de reconstituer le parcours exact de chaque objet depuis sa spoliation jusqu'en 2000, année à laquelle l'institution a décidé, soixante ans après les événements, de restituer les objets. Certains de ces objets ont disparus depuis leur spoliation et seule l'archive conserve leur trace. J'indique, sur le fond gris d'un espace vide, leur absence, par l'inscription de la dénomination adoptée dans l'inventaire. Les mots se substituent à l'objet et rendent sa perte lisible.

Dans Dies ist der Stuhl für den Paten (2001), j'ai travaillé sur la spoliation d'une synagogue en Autriche. Après l'Anschluss de 1938, tous les objets liturgiques de la synagogue de Hohenems avaient été confisqués et revendus. Seule l'archive - une liste précise rédigée lors de la spoliation - me permettait d'en reconstituer le processus. L'unique objet dont j'avais retrouvé une trace visuelle était une double-chaise de circoncision ; sa photographie avait été prise quelques années auparavant par Theodor Harburger, un ethnologue allemand qui s'intéressait aux objets de culte juifs autour du lac de Constance. J'avais temporairement transformé l'espace en une vaste chambre noire, baignée dans une lumière inactinique, contenant un bassin dans lequel flottait un agrandissement de $5 \times 5 \mathrm{~m}$ de cette image. Une voix énumérait tout simplement les objets « disparus ».

Johann Chapoutot. Comment l'art et les images peuvent-ils se saisir d'un tel héritage? Dans ce contexte, la notion de réparation vous parait-elle pertinente?

L'histoire des spoliations nazies, et du traitement juridique de ces spoliations après 1945, peut-elle être riche de leçons pour le sort de collections mises à mal ces dernières décennies (en Irak notamment)?

De quelles manières la question de la spoliation des ceuvres d'art par les nazis se distingue-t-elle de celle du pillage des cuvres d'art "non-occidentales » qui peuplent les collections des musées d'art premier en Occident?

Christoph Zuschlag. Nous ne pouvons pas effacer les crimes du régime nazi, mais nous pouvons et nous avons le devoir de répondre de notre héritage historique. C'est pour cette raison que nous avons la responsabilité morale et éthique de dédommager les victimes des persécutions nazies ou leurs descendants. Si les musées veulent se montrer dignes de leur mission éducative, ils doivent adopter un comportement exemplaire du point de vue éthique en matière de gestion de leurs collections et assumer en toute honnêteté leur provenance et leur histoire - avec toutes les conséquences que cela implique. Le cas de l'État nazi montre à quelles menaces peuvent être exposés l'art et les biens culturels, même lorsqu'ils appartiennent à l'État. Les persécutions et les expropriations perpétrées pour des raisons de « races » font malheureusement partie de l'histoire de l'humanité au même titre que les guerres et le trafic illicite de biens culturels dévoyés en butin de guerre ou encore leur destruction matérielle, comme l'a perpétré l'État islamique en Syrie et en Irak. Il est bien entendu important de prendre en compte et de différencier les différents contextes. Mais il est également nécessaire de tirer des leçons de l'histoire et de s'assurer, par exemple, que le droit international ou les critères éthiques de l'International Council of Museums (ICOM) répondent encore aux exigences actuelles.

On trouve bien des cas de figure différents au cours de la période qui nous occupe, comme il existe une grande diversité de situations selon les différents contextes historiques. On ne peut statuer qu'au cas par cas en enquêtant pour savoir s'il s'agit $d^{\prime}$ 'une soustraction frauduleuse $d^{\prime}$ 'un bien culturel et, si tel est le cas, en déterminant 
les circonstances du vol. Quant aux conséquences à tirer de ces résultats, c'est une toute autre question dont il faut débattre en société et dans la sphère politique. II ne revient pas aux seuls chercheurs d'en décider. En Allemagne, la recherche scientifique sur la provenance des biens culturels restera encore longtemps le principal acteur à mener des recherches autour des œuvres d'art spoliées par les nazis, mais on constate aussi l'apparition de nouveaux débats impliquant à leur tour de nouveaux domaines d'investigations, comme la question des expropriations dans l'ancienne zone d'occupation soviétique et en ex-RDA. La confrontation à I'héritage colonial fait actuellement aussi l'objet de vifs débats (il suffit de penser à la construction du Humboldt Forum à Berlin). La possibilité de restituer des biens culturels dérobés à l'époque coloniale qu'a laissé entrevoir le Président français Macron à Ouagadougou le 28 novembre 2017 pourrait entraîner un changement de paradigme. Dans ce cas aussi, la recherche scientifique sur la provenance des biens culturels doit constituer une base indispensable qui permet d'appréhender la vie de ces objets dans leur contexte historique respectif et de les replacer dans une perspective transnationale et globale.

Emmanuelle Polack. La sémantique est essentielle dans le débat qui nous intéresse. Aussi nous faut-il définir l'action de restituer. Une première définition peut nous être donnée par le dictionnaire : restituer c'est rendre ce qui a été pris ou ce qui est possédé indûment. Pour autant, dès janvier 1945, le professeur Émile Terroine (1882-1974), I'un des premiers acteurs impliqué dans le processus de restitution des biens culturels aux familles juives, en livrait une autre interprétation : « la restitution des biens spoliés est une œuvre de justice et d'humanité dont la signification morale et politique dépasse de beaucoup les valeurs matérielles. Elle doit être, aux yeux de la France et du monde, une des grandes manifestations tangibles du rétablissement du droit et de la légalité républicaine ${ }^{13}$. » En ce sens, la restitution des biens juifs s'entend au sein d'une mission qui, loin de s'attacher à diverses opérations comptables où la valeur numéraire d'une œuvre serait questionnée, se donnerait tous les moyens nécessaires à l'établissement de l'origine de la propriété d'une œuvre ou d'un objet culturel. Et ce afin de réparer un vol légalisé par l'aryanisation économique, qu'il soit issu des ordonnances de l'autorité occupante ou bien des lois de Vichy (fig. 9). L'action de restituer doit être entendue alors comme une volonté d'apporter compréhension et empathie aux familles des victimes des persécutions raciales. Ce n'est que si elle est appuyée sur la reconnaissance des exactions perpétrées lors de la Seconde Guerre mondiale que la restitution peut apparaître véritablement réparatrice. Il faut toujours garder à l'esprit que la spoliation des biens et objets culturels juifs s'inscrit dans un continuum qui va de la stigmatisation d'une partie d'une population, à partir de son recensement, en passant par son éradication de l'économie nationale, son pillage, sa spoliation et sa déportation, jusqu'à son extermination, et qu'elle participe en ce sens de l'unicité de la Shoah.
9. Catalogue de vente Biens israélites [...] dont la vente aux enchères publiques aura lieu [à I']Hôtel Drouot, salle n¹, le lundi 13 décembre 1943

Paris, bibliothèque de I'INHA collections Jacques Doucet, NUM CVP01216_19431213 (VP 1943/443).

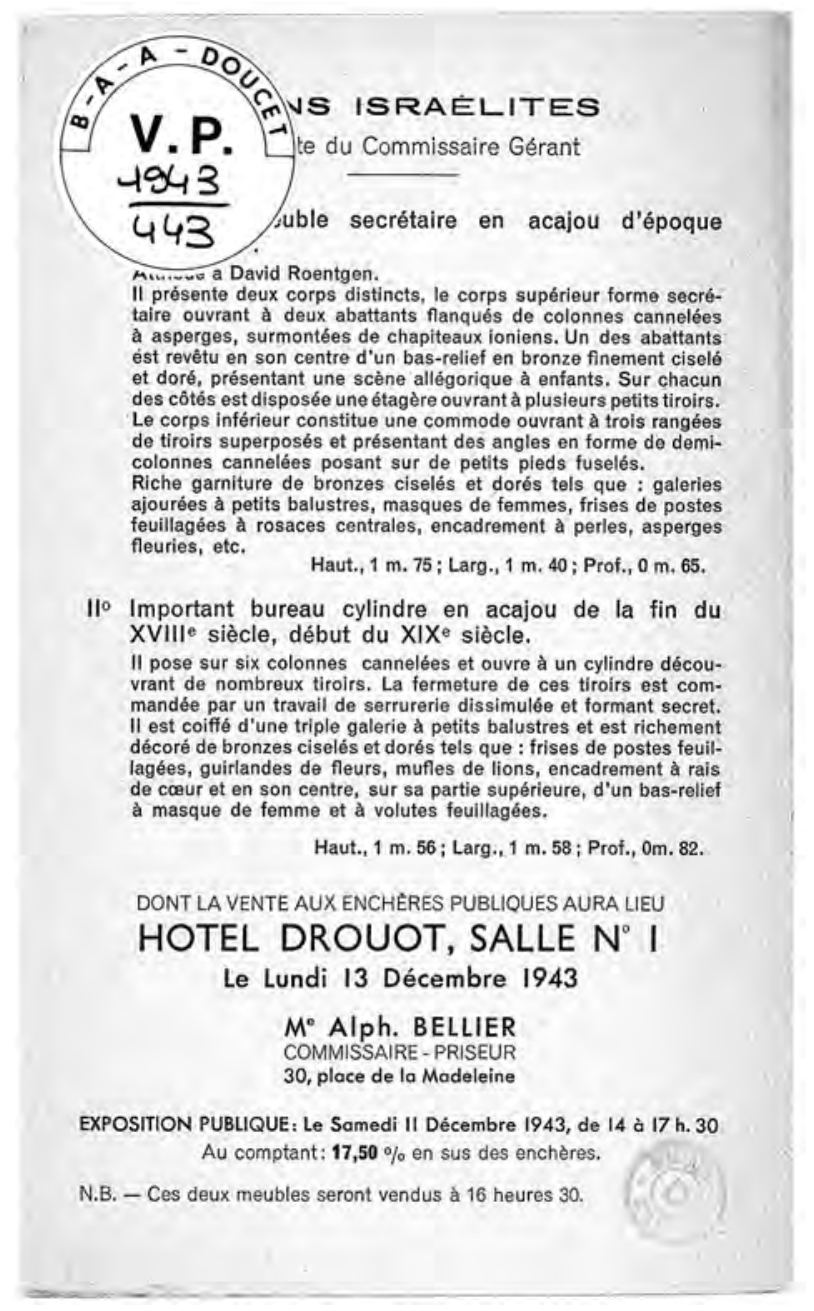




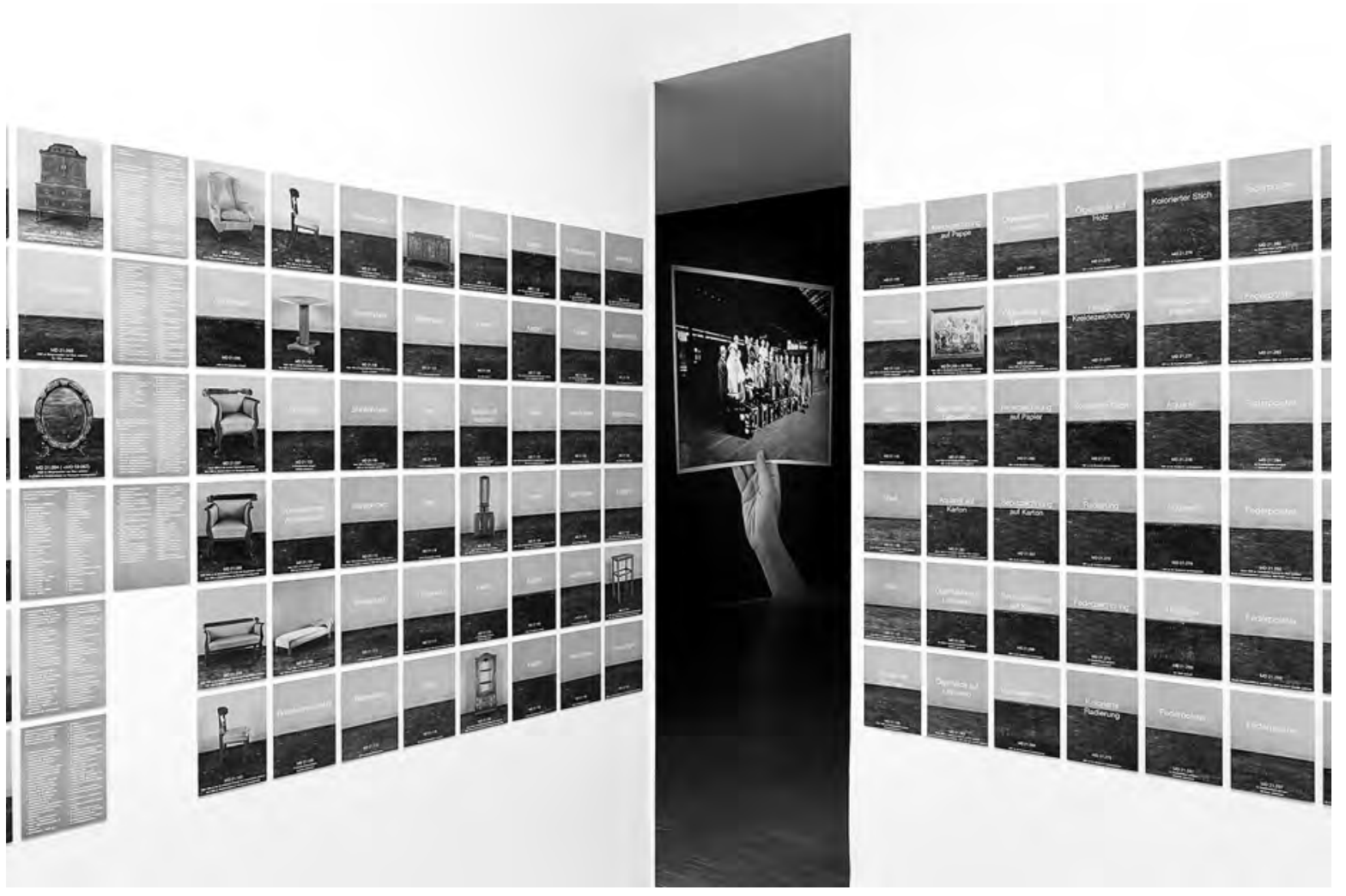

10. Arno Gisinger, Invent arisiert, 2000, exposé au Centre Pompidou, en 2014, Paris. Photographie Romain Darnaud.
C'est seulement si cet esprit de justice et d'humanité prévaut dans la cohérence de ce travail de mémoire que l'expérience de la restitution des biens juifs peut tendre vers I'universalité.

Arno Gisinger. Je pense qu'il faut être très précis dans ces questions, justement pour les faire résonner de manière plus générale. À propos de la question spécifique qui nous concerne ici, il faut, comme Christoph Zuschlag le signale à juste titre, distinguer "spoliation » et "Kunstraub ». II faut aussi faire la distinction entre ce qu'il se passe en Allemagne, en Autriche et dans les différents pays occupés. Le terme « réparation » peut devenir très vite très complexe, car il faut déjà bien identifier - pour reprendre les termes de Raoul Hilberg - les victimes, les persécuteurs et les " témoins» (bystanders) d'un tel processus.

En allemand, nous avons le mot Wiedergutmachung, difficilement traduisible. Il signifie d'abord un « dédommagement matériel » mais aussi, au sens psychologique du terme, une sorte de "réparation morale rétroactive ». J'avoue avoir du mal avec cette seconde signification, car pour moi les deux seules façons d'affronter les crimes du passé sont la justice et l'histoire. Au fameux « devoir de mémoire » je préfère toujours un "droit de savoir ». Je ne pense pas que l'art puisse "réparer » quoi que ce soit. Mais il peut complexifier notre vision de l'histoire, nous sensibiliser, nous alerter et nous responsabiliser en tant que regardeurs et citoyens.

J'expliquais précédemment qu'il fallait replacer la spoliation des œuvres d'art dans le contexte général de la spoliation. Pour conclure, je dirais qu'il faudrait également la replacer dans le contexte plus large des relations que les œuvres d'art entretiennent avec les lieux géographiques, institutionnels et personnels. En ce sens, la notion de 
« translocation des œuvres » développée par Bénédicte Savoy me paraît un excellent mode opératoire pour éviter tout communautarisme et eurocentrisme dans la question de la spoliation.

Johann Chapoutot. À ce propos, pensez-vous qu'il y ait des lieux plus propices à l'exposition de travaux sur les spoliations? Quelle importance le lieu d'exposition a-t-il dans la réception de ce type d'cuvres?

Arno Gisinger. Les différentes strates formelles et institutionnelles d'un lieu d'exposition jouent toujours un rôle fondamental dans la réception des œuvres. Je ne pense pas qu'il y ait un " lieu idéal » pour des travaux sur la spoliation. Par contre, les artistes, les chercheurs et les commissaires d'exposition doivent prendre en compte le contexte matériel et esthétique du lieu de façon précise. J'ai expérimenté ce phénomène de déplacement de la réception avec le travail Invent arisiert déjà cité. À l'origine, je I'avais conçu, réalisé et montré au Mobiliendepot à Vienne. Il y avait une sorte d'unité de lieu - par ailleurs assez troublante pour l'institution même - entre le stockage et le prêt de ces meubles depuis 1938, mes prises de vue en 1999 et l'exposition des images en 2000. Le travail s'adressait tout d'abord à un public autrichien directement concerné par cette question. Des années plus tard, j'ai eu l'occasion de montrer le même travail à Lyon, au Centre d'histoire de la résistance et de la déportation, dans un contexte français et dans un lieu destiné à l'histoire. La réception n'était pas du tout la même. En 2014, Invent arisiert a finalement été présenté au Centre Pompidou (fig. 10), en résonance avec d'autres œuvres, dans le cadre du Nouveau Festival, sous le titre Éblouis par l'oubli. Je ne peux pas vous dire lequel des trois lieux était le plus " propice » mais les trois ensembles élargissaient les potentiels champs de réception de façon très surprenante.

La contribution de Christoph Zuschlag

a été traduite de l'allemand

par Bérénice Zunino. 
Note de la rédaction. Dans ce débat, comme dans le débat suivant, au sujet de la constitution et de la restitution des collections "non-occidentales » (p. 37-70), le comité scientifique de la revue a souhaité aborder la question des restitutions en donnant la parole aux principaux acteurs concernés, historiens aussi bien qu'historiens de l'art, juristes, artistes et conservateurs de musée.

1. Éric Michaud, Un art de l'éternité. L'image et le temps du national-socialisme, Paris, Gallimard ("Le temps des Images »), 1996.

2. Christoph Zuschlag, "Entartete Kunst ». Ausstellungsstrategien im Nazi-Deutschland, Worms, Wernersche Verlagsgesellschaft, 1995 ; Idem, " 75 Jahre Ausstellung "Entartete Kunst" », dans Matthias Wemhoff, Meike Hoffmann, Dieter Scholz (dir.), Der Berliner Skulpturenfund. "Entartete Kunst " im Bombenschutt, Entdeckung - Deutung - Perspektive, livret d'accompagnement de l'exposition (Berlin, musée de la Préhistoire et de la Protohistoire, Neues Museum / Hambourg, Museum für Kunst und Gewerbe / Munich, Neue Pinakothek, 2010-2013), et rassemblant les contributions du colloque (Berlin, 15-16 mars 2012), Ratisbonne, Scnell \& Steiner, 2012, p. 37-51.

3. Stephanie Barron, Degenerate Art, The Fate of the Avant-Garde in Nazi Germany, cat. exp. (Los Angeles, County Museum of Art / Art Institute of Chicago, 1991), Los Angeles / New York, Abrams / Los Angeles County Museum of Art, 1991, p. 402.

4. Johanna Linsler, Mica Gherghescu, Didier Schulmann (dir.), Les Sources au travail : les spoliations d'œuvres d'art par les nazis, 1933-2015, Journal de l'université d'été de la bibliothèque Kandinsky, $\mathrm{n}^{\circ} 2$ 2, Paris, Musée national d'art moderne/CCl, bibliothèque Kandinsky, 2015.

5. Carl-Heinz Heuer, « Die eigentumsrechtliche Problematik der "Entarteten Kunst" ", dans Meike Hoffmann, Andreas Hüneke (dir.), Auf den Spuren der verlorenen Moderne. 10 Jahre Forschungsstelle "Entartete Kunst » am Kunsthistorischen Institut der Freien Universität Berlin, actes du colloque (Berlin, Kunsthistorischen Institut der Freien Universität, 2013), Berlin 2013, p. 10-14, ici p. 12-13.

6. MNR pour «Musées Nationaux Récupérations".

7. En décembre 1998 à Washington, 44 États et 13 ONG signaient une déclaration déterminant les règles de restitution des biens culturels volés par les nazis.

8. Constantin Goschler, « Zwei Wellen der Restitution: die Rückgabe jüdischen Eigentums nach 1945 und 1990 ", dans Inka Bertz, Michael Dorrmann (dir.), Raub und Restitution. Kulturgut aus jüdischem Besitz von 1933 bis heute, cat. exp. (Berlin, Jüdisches Museum / Francfort-sur-le-Main, Jüdisches Museum, 2008-2009), Göttingen / Berlin, Wallstein / Jüdisches Museum, 2008, p. 30-45, ici p. 30.
9. http://www.culture.gouv.fr/documentation/mnr/.

10. Décret n99-778 du 10 septembre 1999 « instituant une commission pour l'indemnisation des victimes de spoliations intervenues du fait des législations antisémites en vigueur pendant l'Occupation ».

11. Un outil spécifique est à disposition des membres du groupe de travail et permet de faire des recherches en plein texte dans les catalogues de vente numérisés et océrisés de la bibliothèque de I'INHA pour la période 1914-1950, grâce à un travail conduit par le département des Études et de la Recherche de I'INHA.

12. Frits Lugt, Les marques de collections de dessins \& d'estampes : avec des notices historiques sur les collectionneurs, les collections, les ventes,..., Amsterdam, Vereenigde drukkerijen, 1921 .

13. Claire Andrieu (dir.), La spoliation financière, Paris, La documentation française, 2000. 


\section{Un programme de l'Institut national d'histoire de l'art}

Depuis le début de l'année 2017, I'INHA a lancé le programme « Répertoire des acteurs du marché de l'art en France pendant l'occupation allemande », en partenariat avec la Technische Universität de Berlin et en coopération avec le Deutsches Zentrum Kulturgutverluste de Magdebourg. Ce programme vise à étudier et à répertorier l'ensemble des acteurs (marchands d'art, galeristes, courtiers, experts, antiquaires, commissairespriseurs, transporteurs, photographes, historiens de l'art, personnel des musées, artistes, collectionneurs, amateurs, victimes, intermédiaires en tout genre...) qui ont été partie prenante des échanges artistiques et commerciaux entre la France et l'Allemagne entre 1940 et 1945 . Il veut documenter et reconstituer, de la manière la plus rigoureuse et la plus objective, les parcours des hommes et des œuvres, et mettre en évidence les circulations et les réseaux.

Ce répertoire prendra principalement la forme d'une base de données, en accès libre et gratuit, avec des entrées individuelles qui permettront de procéder à des croisements et à des vérifications systématiques des informations. Celles-ci seront strictement factuelles et fondées sur des recherches prioritairement menées dans les archives allemandes et françaises, voire de tout autre pays (États-Unis, Belgique, Hollande, Autriche, Suisse, Russie, etc.) dont la prise en compte apparaîtrait nécessaire.

Ce programme s'inscrit dans la continuité de la mise en ligne par l'INHA, depuis 2012, des catalogues de vente parisiens de cette période, à partir d'une recherche et d'une numérisation menée en collaboration avec le Service des musées de France et financée avec l'aide de la Fondation pour la mémoire de la Shoah. Dans une perspective à la fois plus ample, plus systématique et plus explicitement centrée sur les spoliations dont les principales victimes furent les Juifs, il veut offrir un outil fiable et scientifique à l'ensemble des utilisateurs, citoyens, chercheurs ou professionnels du monde de l'art, préoccupés de vérifier la provenance d'une œuvre, que celle-ci se trouve dans les collections publiques, en mains privées ou sur le marché, et de mieux comprendre ce sujet encore brûlant.

Ce programme binational, conduit par Inès Rotermund-Reynard depuis janvier 2018 pour l'INHA (sous la direction d'Ariane James-Sarazin) et par Elisabeth Furtwängler pour la Technische Universität de Berlin (sous celle de Bénédicte Savoy), s'inscrit également dans une dynamique internationale de recherche sur l'histoire des spoliations pendant la Seconde Guerre mondiale, marquée par la multiplication récente des colloques et des journées d'études sur cette question (notamment le colloque «Raub \& Handel. Der französische Kunstmarkt unter deutscher Besatzung (1940-1944) » [Spoliation et trafic. Le marché de l'art français sous I'occupation allemande (19401944)], organisé par le Deutsches Zentrum Kulturgutverluste à Bonn, les 30 novembre et $1^{\text {er }}$ décembre 2017). Mais il entend aussi dépasser les controverses et les limites des travaux menés sur sa dimension française. Dès 1993, I'historienne de l'art Laurence Bertrand Dorléac présentait I'ampleur et la spécificité du trafic et du commerce des objets d'art entre 1940 et 1945 : «Quinze jours seulement après I'armistice commençait donc une formidable opération de pillage des œuvres d'art selon la loi du plus fort. [...] Après la guerre, [...] les dossiers n'en établissaient pas moins I'action de brigandage de certains Français, marchands intermédiaires surtout, qui, transformés en larrons du "second marché", s'étaient à contrecœur contentés des restes, voyant 
filer outre-Rhin les milliers d'œuvres de collectionneurs juifs dépossédés » (L'art de la défaite. 1940-1944, Paris, Seuil, 1993, p. 20-21). En 1995, la traduction française de l'ouvrage pionnier de la chercheuse indépendante Lynn Nicholas, Le pillage en Europe. Les œeuvres d'art volées par les nazis (Paris, Seuil), paru l'année précédente en anglais (The Rape of Europa: The Fate of Europe's Treasures in the Third Reich and the Second World War, New York, Knopf, 1994), et la publication de l'enquête journalistique d'Hector Feliciano, Le musée disparu. Enquête sur le pillage d'œuvres d'art en France par les nazis (Paris, Austral), alertaient le grand public, au prix de simplifications hâtives mais aussi de véritables difficultés sur le terrain, sur l'ampleur des spoliations et le caractère partiel des restitutions. La mise en place de la commission Mattéoli en 1997 et la publication sous son égide d'un rapport rédigé par Didier Schulmann et Isabelle Le Masne de Chermont, Le Pillage de l'art en France pendant l'Occupation et la situation des 2000 œuvres confiées aux Musées nationaux (Paris, La Documentation française, 2000), allaient permettre certaines avancées, notamment grâce à l'action de la Commission d'indemnisation des victimes de spoliation (CIVS). En menant une mission sur les œuvres au passé flou qui aboutit à la publication du livre " Si les tableaux pouvaient parler "... Le traitement politique et médiatique des retours d'œuvres $d$ 'art pillées et spoliées par les nazis (France 1945-2008) (Rennes, Presses universitaires de Rennes, 2013), I'historienne et sénatrice Corinne Bouchoux anticipe et accompagne une forte demande des autorités gouvernementales sur ce sujet, qui conduit certains musées, d'abord en province (à Angers en 2014, à Rennes en 2015) puis à Paris, à mener des politiques volontaristes d'identification, de documentation et de mise en valeur des œuvres inscrites comme MNR. À la même époque, est instituée une formation spécifique des élèves de l'Institut national du patrimoine et des étudiants de l'École du Louvre, tandis que le hall d'accueil de la galerie Colbert, l'un des deux sites de l'INHA, est symboliquement baptisé du nom de Rose Valland.

Pourtant, plus de soixante-dix ans après la fin de la Seconde Guerre mondiale, nos connaissances sur les transferts, les trafics et les spoliations d'œuvres d'art générés par l'occupation allemande en France ou permis par elle (avec la complicité du régime de Vichy) sont encore trop lacunaires. Si de nombreuses enquêtes individuelles ont été menées ces dernières années, après la période de relatif désintérêt pour cette question qui a suivi I'action conduite notamment par Rose Valland dès la fin de la guerre, si elles ont donné lieu à certaines restitutions, une identification exhaustive des différents acteurs du marché de l'art de cette époque, des opérations qu'ils ont effectuées, des œuvres qui sont passées entre leurs mains, devient urgente, notamment afin de fournir un fondement stable à la documentation des œuvres et aux recherches concernant leur histoire et leur provenance.

Éric de Chassey, directeur général de I'INHA 